\title{
A Bivariate Compound Dynamic Contagion Process for Cyber Insurance
}

\author{
Jiwook Jang \\ Department of Actuarial Studies \& Business Analytics, Macquarie Business School, \\ Macquarie University, Sydney NSW 2109, Australia, E-mail: jiwook.jang@mq.edu.au \\ Rosy Oh
}

Institute of Mathematical Sciences, Ewha Womans University, Seoul, 03760, Korea, E-mail: rosy.oh5@gmail.com

\begin{abstract}
As corporates and governments become more digital, they become vulnerable to various forms of cyber attack. Cyber insurance products have been used as risk management tools, yet their pricing does not reflect actual risk, including that of multiple, catastrophic and contagious losses. For the modelling of aggregate losses from cyber events, in this paper we introduce a bivariate compound dynamic contagion process, where the bivariate dynamic contagion process is a point process that includes both externally excited joint jumps, which are distributed according to a shot noise Cox process and two separate self-excited jumps, which are distributed according to the branching structure of a Hawkes process with an exponential fertility rate, respectively. We analyse the theoretical distributional properties for these processes systematically, based on the piecewise deterministic Markov process developed by Davis (1984) and the univariate dynamic contagion process theory developed by Dassios and Zhao (2011). The analytic expression of the Laplace transform of the compound process and its moments are presented, which have the potential to be applicable to a variety of problems in credit, insurance, market and other operational risks. As an application of this process, we provide insurance premium calculations based on its moments. Numerical examples show that this compound process can be used for the modelling of aggregate losses from cyber events. We also provide the simulation algorithm for statistical analysis, further business applications and research.
\end{abstract}

Keywords: Aggregate losses from cyber events; Contagion risk; Bivariate compound dynamic contagion process; Hawkes process; Piecewise deterministic Markov process; Martingale methodology; Insurance premium

\section{Introduction}

Due to the digitalisation of business and economic activities via the Internet of Things (IoT), cloud computing, mobile and other innovative technologies, cyber risk is inherent and extreme. Cyber risks refer to any risk of financial loss, disruption to operations, or damage to the reputation of an organisation due to failure of its information technology (IT) systems, as defined by the Institute of Risk Management (IRM). Financial losses from malicious cyber activities result from IT security/data/digital assets recovery, liability in respect of identity 
theft and data breaches, reputation/brand damage, legal liability, cyber extortion, regulatory defence and penalties coverage and business interruption.

The frequency of malicious cyber activities is rapidly increasing, with the scope and nature dependent on an organisation's industry, size and location. According to a 2016 Allianz survey, cyber risk is the top long-term risk to business and currently a top-three global business risk. It is therefore critical that corporations and governments focus on IT and network security enhancement. Unless public and private sector organisations have effective cyber security plans and strategies in place, and tools to manage and mitigate losses from cyber risks, cyber events have the potential to affect their business significantly, possibly damaging hard-earned reputations irreparably.

Insurance has served to mitigate liability since the 17th century, after the Great Fire of London in 1666. As part of a cyber risk mitigation strategy, cyber insurance can be purchased by organisations to cover economic and financial losses occurring from cyber incidents. Since the widespread Y2K concerns raised the profile of the possible security vulnerabilities of digitalisation, the cyber insurance industry has grown to a total annual premium of $\$ 2.5$ billion, and the market is expected to reach $\$ 20$ billion by 2025 globally. However, due to the complexity of cyber incidents, i.e. multiple, catastrophic and contagious losses, it is difficult for insurers to price cyber insurance products accurately. Inaccurate pricing could have severe market effects in the event of a significant claim.

To date however there has been little theoretical work done on developing acceptable cyber insurance pricing models. Also due to the complexity of cyber risks, the previous studies (Mukhopadhyay et al. 2006; Herath and Herath 2011and Xu and Hua 2017) do not provide a suitable framework to measure cyber risks as they have not accounted for future cyber attacks dynamically. Also traditionally insurance claim modelling has used homogeneous/non-homogeneous Poisson processes as a claim arrival process. However, for cyber events, the assumption that resulting claims occur in terms of the Poisson process is inadequate due to its deterministic intensity. Therefore, an alternative point process needs to be used to predict claim arrivals from cyber incidents.

To this effect, we introduce a bivariate compound dynamic contagion process (BCDCP) for the modelling of aggregate losses from cyber events, where the bivariate dynamic contagion process (BDCP) is a point process which has both externally excited joint jumps, which are distributed according to a shot noise Cox process and two separate self-excited jumps, which are Hawkes processes. Since Hawkes (1971a, 1971b) and Hawkes and Oakes (1974) introduced a self-exciting point process, the applications and modelling of Hawkes processes in finance and insurance can be found in Chavez-Demoulin et al. (2005), McNeil et al. (2005), Bauwens and Hautsch (2009), Bowsher (2007), Errais et al. (2010), Stabile and Torrisi (2010), Embrechts et al. (2011), Giesecke and Kim (2011) and Aït-Sahalia et al. (2014, 2015).

Dassios and Zhao (2011) introduced a dynamic contagion process, which is a generalisation of the externally excited Cox process with shot noise intensity and the self-excited Hawkes process applying to credit risk. Dassios and Zhao (2012) also examined infinite horizon ruin probability with its Monte Carlo simulation using this process as the claim arrival process. Dassios and Zhao (2017a) extended this process with diffusion component to calculate the default probability and to price defaultable zero-coupon bonds. We have found dynamic contagion processes to be flexible and realistic in modelling claims with contagion.

These aforementioned papers are neither the bivariate dynamic contagion models nor the 
compound models. In contrast we extend it further to quantify aggregate losses from cyber events using a bivariate compound dynamic contagion process as they are multiple, catastrophic and contagious losses. Biener et al. (2015) emphasised that one of characteristics of cyber risk is highly interrelated losses, and modelling cyber risk would be a great deal of promise to test them when enough cyber loss data become available.

Bivariate modelling with self-exciting Hawkes processes can be noticed in Jang and Dassios (2013), where they introduced a bivariate shot noise self-exciting process that can be used for the modelling of catastrophic losses. Dong (2014) examined the stationarity of bivariate dynamic contagion processes including the cross-exciting contagion effect in his doctoral thesis. Applications and modelling of multivariate Hawkes process in high-frequency limit order book data can be found in Rombaldi et al. (2017) and Lu and Abergel (2018). Yang et al. (2018) investigated the interactions between market return events and investor sentiment using a multivariate Hawkes process.

Compound modelling with univariate self-exciting Hawkes processes can be noticed in Dassios and Zhao (2017b), where they developed the algorithms for a generalised self-exciting point process with CIR-type intensities. Gao et al. (2018) applied the joint Laplace transform of the classical Hawkes process and its compound process in dark pool trading, which do not display bid and ask quotes to the public.

This project develops a new model for pricing cyber risk using a BCDCP, which accommodate the interdependence dynamics of IT system and the frequency and impact of cyber events. Our research offers a new framework to enable insurance companies to price cyber insurance policies accommodating clustering of losses.

This paper is structured as follows. In Section 2, we provide a mathematical definition of the BCDCP and the BDCP, respectively via the stochastic intensity representation adopted the one used by Dassios and Zhao (2011) and the algorithm for simulating these processes in Section 5. In Section 3, we analyse these processes systematically for their theoretical distributional properties, based on the piecewise deterministic Markov process theory developed by Davis (1984), and the martingale methodology used by Dassios and Jang (2003). The joint moment of two processes, its covariance and linear correlation are derived in Section 4, where for simplicity, we use the case for the stationary distribution of the intensity processes. As an application of this process, we provide cyber insurance premium calculations based on these quantities in Section 5. Section 6 concludes the paper.

\section{Definition}

In this section, we have a mathematical definition for the BCDCP in Definition 2.2. Before that, let us have a mathematical definition for the BDCP in Definition 2.1 via the stochastic intensity representation adopted the one used by Dassios and Zhao (2017). For an alternative definition for this process, we refer you Dassios and Zhao (2011), Jang and Dassios (2013) and Dong (2014), where they gave as a cluster process representation for the univariate dynamic contagion process, the bivariate shot noise self-exciting process and the bivariate dynamic contagion process, respectively.

Definition 2.1 (Bivariate dynamic contagion process). Bivariate dynamic contagion process is a point process $\left(\begin{array}{c}N_{t}^{(1)} \\ N_{t}^{(2)}\end{array}\right)_{t>0}=\left(\begin{array}{c}\sum_{j \geq 1} \mathbb{I}\left(T_{2, j} \leq t\right)_{j=1,2, \cdots} \\ \sum_{k \geq 1} \mathbb{I}\left(T_{2, k} \leq t\right)_{k=1,2, \cdots}\end{array}\right)$ with the non- 
negative $\Im_{t}$-stochastic bivariate intensity process $\left(\begin{array}{c}\lambda_{t}^{(1)} \\ \lambda_{t}^{(2)}\end{array}\right)$, i.e.

$$
\begin{aligned}
\lambda_{t}^{(1)}= & a^{(1)}+\left(\lambda_{0}^{(1)}-a^{(1)}\right) e^{-\delta^{(1)} t}+\sum_{i \geq 1} X_{i}^{(1)} e^{-\delta^{(1)}\left(t-T_{1, i}\right)} \mathbb{I}\left(T_{1, i} \leq t\right) \\
& +\sum_{j \geq 1} Y_{j} e^{-\delta^{(1)}\left(t-T_{2, j}\right)} \mathbb{I}\left(T_{2, j} \leq t\right), \\
\lambda_{t}^{(2)}= & a^{(2)}+\left(\lambda_{0}^{(2)}-a^{(2)}\right) e^{-\delta^{(2)} t}+\sum_{i \geq 1} X_{i}^{(2)} e^{-\delta^{(2)}\left(t-T_{1, i}\right)} \mathbb{I}\left(T_{1, i} \leq t\right) \\
& +\sum_{k \geq 1} Z_{k} e^{-\delta^{(2)}\left(t-T_{2, k}\right)} \mathbb{I}\left(T_{2, k} \leq t\right),
\end{aligned}
$$

where

- $\left\{\Im_{t}\right\}_{t \geq 0}$ is a history of the joint process $\left(\begin{array}{c}N_{t}^{(1)} \\ N_{t}^{(2)}\end{array}\right)$, with respect to which $\left\{\begin{array}{l}\lambda_{t}^{(1)} \\ \lambda_{t}^{(2)}\end{array}\right\}_{t \geq 0}$ is adapted;

- $\lambda_{0}^{(d)}>0$ is the initial intensity at time $t=0$, where $d=1,2$;

- $a^{(d)} \geq 0$ is the constant mean-reverting level;

- $\delta^{(d)}>0$ is the constant mean-reverting rate;

- $\left\{X_{i}^{(1)}, X_{i}^{(2)}\right\}_{i=1,2, \cdots}$ is a sequence of i.i.d. positive externally-excited joint jumps with distribution $F\left(x^{(1)}, x^{(2)}\right), x^{(1)}>0, x^{(2)}>0$, where margins are $F_{X^{(1)}}$ and $F_{X^{(2)}}$ at the corresponding random times $\left\{T_{1, i}\right\}_{i=1,2, \ldots}$ following a Poisson process $M_{t}$ with constant rate $\rho>0$, and $\mathbb{I}$ is the indicator function.

- $\left\{Y_{j}\right\}_{j=1,2, \ldots}$ is a sequence of i.i.d. positive self-excited jumps with distribution function $G(y), y>0$, at the corresponding random times $\left\{T_{2, j}\right\}_{j=1,2, \cdots}$.

- $\left\{Z_{k}\right\}_{k=1,2, \ldots}$ is another sequence of $i . i . d$. positive self-excited jumps with distribution function $H(z), z>0$, at the corresponding random times $\left\{T_{2, k}\right\}_{k=1,2, \cdots}$.

- $\left\{X_{i}^{(1)}, X_{i}^{(2)}\right\}_{i=1,2, \cdots},\left\{Y_{j}\right\}_{j=1,2, \cdots},\left\{Z_{k}\right\}_{k=1,2, \cdots},\left\{T_{1, i}\right\}_{i=1,2, \ldots},\left\{T_{2, j}\right\}_{j=1,2, \cdots}$ and $\left\{T_{2, k}\right\}_{k=1,2, \ldots}$ are assumed to be independent of each other.

The bivariate compound model we consider has the following structure:

$$
\begin{aligned}
L_{t}^{(1)} & =\sum_{j \geq 1} \Xi_{j}^{(1)} \mathbb{I}\left(T_{2, j} \leq t\right), \\
L_{t}^{(2)} & =\sum_{k \geq 1} \Xi_{k}^{(2)} \mathbb{I}\left(T_{2, k} \leq t\right),
\end{aligned}
$$


where $L_{t}^{(d)}$ is the total amount of claims/losses arising from risk type $d=1,2$ and $N_{t}^{(d)}$ is the number of points (i.e. claims/losses) up to time $t$. The random variables $\Xi_{j}^{(1)}$ and $\Xi_{k}^{(2)}$ denote the individual claim/loss amounts, where we assume that they are independent identically distributed with distributions $J_{Y^{(1)}}$ and $K_{Y^{(2)}}$, respectively. Our intensity processes for $N_{t}^{(1)}$ and $N_{t}^{(2)}$ are modelled by jump processes, which are in the form of (2.1).

Definition 2.2 (Bivariate compound dynamic contagion process). Bivariate compound dynamic contagion process is a compound point process $\left(\begin{array}{c}L_{t}^{(1)} \\ L_{t}^{(2)}\end{array}\right)_{t>0}=$ $\left(\begin{array}{c}\sum_{j \geq 1} \Xi_{j}^{(1)} \mathbb{I}\left(T_{2, j} \leq t\right)_{j=1,2, \cdots} \\ \sum_{k \geq 1} \Xi_{k}^{(2)} \mathbb{I}\left(T_{2, k} \leq t\right)_{k=1,2, \cdots}\end{array}\right)$ with the non-negative $\Im_{t}$-stochastic bivariate intensity pro$\operatorname{cess}\left(\begin{array}{c}\lambda_{t}^{(1)} \\ \lambda_{t}^{(2)}\end{array}\right)$ which is in the form of $(2.1)$, where

- $\left\{\Xi_{j}^{(1)}\right\}_{j=1,2, \cdots}$ is a sequence of $i . i . d$. positive individual claim/loss amounts from risk type $d=1$ with distribution function $J\left(\xi^{(1)}\right), \xi^{(1)}>0$, at the corresponding random times $\left\{T_{2, j}\right\}_{j=1,2, \cdots}$.

- $\left\{\Xi_{k}^{(2)}\right\}_{k=1,2, \cdots}$ is another sequence of i.i.d. positive individual claim/loss amounts from risk type $d=2$ with distribution function $K\left(\xi^{(2)}\right), \xi^{(2)}>0$, at the corresponding random times $\left\{T_{2, k}\right\}_{k=1,2, \cdots}$.

• $\left\{X_{i}^{(1)}, X_{i}^{(2)}\right\}_{i=1,2, \cdots},\left\{Y_{j}\right\}_{j=1,2, \cdots},\left\{Z_{k}\right\}_{k=1,2, \cdots},\left\{\Xi_{j}^{(1)}\right\}_{j=1,2, \cdots},\left\{\Xi_{k}^{(1)}\right\}_{k=1,2, \cdots},\left\{T_{1, i}\right\}_{i=1,2, \cdots}$ $\left\{T_{2, j}\right\}_{j=1,2, \ldots}$ and $\left\{T_{2, k}\right\}_{k=1,2, \ldots}$ are assumed to be independent of each other.

The joint process of $\left\{\left(\begin{array}{c}\lambda_{t}^{(1)} \\ \lambda_{t}^{(2)}\end{array}\right),\left(\begin{array}{c}N_{t}^{(1)} \\ N_{t}^{(2)}\end{array}\right),\left(\begin{array}{c}L_{t}^{(1)} \\ L_{t}^{(2)}\end{array}\right)\right\}_{t \geq 0} \quad$ is a Markov process in the state space $\mathbb{R}^{+} \times \mathbb{N}_{0} \times \mathbb{R}_{0}^{+}$. With the aid of piecewise deterministic Markov process theory and using the results in Davis (1984), the infinitesimal generator of the bivariate compound dynamic contagion process $\left(\lambda_{t}^{(1)}, N_{t}^{(1)}, L_{t}^{(1)}, \lambda_{t}^{(2)}, N_{t}^{(2)}, L_{t}^{(2)}, t\right)$ acting on a function $f\left(\lambda^{(1)}, n^{(1)}, l^{(1)}, \lambda^{(2)}, n^{(2)}, l^{(2)}, t\right)$ within its domain $\mathcal{D}(\mathcal{A})$ is given by 


$$
\begin{aligned}
& \mathcal{A} f\left(\lambda^{(1)}, n^{(1)}, l^{(1)}, \lambda^{(2)}, n^{(2)}, l^{(2)}, t\right) \\
& =\frac{\partial f}{\partial t}+\delta^{(1)}\left(a^{(1)}-\lambda^{(1)}\right) \frac{\partial f}{\partial \lambda^{(1)}}+\delta^{(2)}\left(a^{(2)}-\lambda^{(2)}\right) \frac{\partial f}{\partial \lambda^{(2)}} \\
& +\lambda^{(1)}\left[\begin{array}{c}
\int_{0}^{\infty} \int_{0}^{\infty} f\left(\lambda^{(1)}+y, n^{(1)}+1, l^{(1)}+\xi^{(1)}, \lambda^{(2)}, n^{(2)}, l^{(2)}, t\right) d G(y) d J\left(\xi^{(1)}\right) \\
-f\left(\lambda^{(1)}, n^{(1)}, l^{(1)}, \lambda^{(2)}, n^{(2)}, l^{(2)}, t\right)
\end{array}\right] \\
& +\lambda^{(2)}\left[\begin{array}{c}
\int_{0}^{\infty} \int_{0}^{\infty} f\left(\lambda^{(1)}, n^{(1)}, l^{(1)}, \lambda^{(2)}+z, n^{(2)}+1, l^{(2)}+\xi^{(2)}, t\right) d H(z) d K\left(\xi^{(2)}\right) \\
-f\left(\lambda^{(1)}, n^{(1)}, l^{(1)}, \lambda^{(2)}, n^{(2)}, l^{(2)}, t\right)
\end{array}\right] \\
& +\rho\left[\begin{array}{c}
\int_{0}^{\infty} \int_{0}^{\infty} f\left(\lambda^{(1)}+x^{(1)}, n^{(1)}, l^{(1)}, \lambda^{(2)}+x^{(2)}, n^{(2)}, l^{(2)}, t\right) d F_{X^{(1)}, X^{(2)}}\left(x^{(1)}, x^{(2)}\right) \\
-f\left(\lambda^{(1)}, n^{(1)}, l^{(1)}, \lambda^{(2)}, n^{(2)}, l^{(2)}, t\right)
\end{array}\right],
\end{aligned}
$$

where $\mathcal{D}(\mathcal{A})$ is the domain of the generator $\mathcal{A}$ such that $f\left(\lambda^{(1)}, n^{(1)}, l^{(1)}, \lambda^{(2)}, n^{(2)}, l^{(2)}, t\right)$ is differentiable with respect to $\lambda^{(1)}, \lambda^{(2)}$ and $t$ for all $\lambda^{(1)}, \lambda^{(2)}$ and $t$, and

$$
\begin{aligned}
& \left|\int_{0}^{\infty} \int_{0}^{\infty} f\left(\cdot, \lambda^{(1)}+y, n^{(1)}+1, l^{(1)}+\xi^{(1)}, \cdot\right) d G(y) d J\left(\xi^{(1)}\right)-f\left(\cdot, \lambda^{(1)}, n^{(1)}, l^{(1)}, \cdot\right)\right|<\infty, \\
& \left|\int_{0}^{\infty} \int_{0}^{\infty} f\left(\cdot, \lambda^{(2)}+z, n^{(2)}+1, l^{(2)}+\xi^{(2)}, \cdot\right) d H(z) d K\left(\xi^{(2)}\right)-f\left(\cdot, \lambda^{(2)}, n^{(2)}, l^{(2)}, \cdot\right)\right|<\infty, \\
& \left|\int_{0}^{\infty} \int_{0}^{\infty} f\left(\cdot, \lambda^{(1)}+x^{(1)}, \lambda^{(2)}+x^{(2)}, \cdot\right) d F\left(x^{(1)}, x^{(2)}\right)-f\left(\cdot, \lambda^{(1)}+x^{(1)}, \lambda^{(2)}+x^{(2)}, \cdot\right)\right|<\infty .
\end{aligned}
$$

\section{Bivariate Compound Dynamic Contagion Process}

In this section, we derive the joint Laplace transform of the process $\left(L_{T}^{(1)}, L_{T}^{(2)}\right)$ in Theorem 3.4, for which we start with Theorem 3.1. Theorem 3.1 leads to the key results of the paper as we also derive the joint probability generating function of the process $\left(N_{T}^{(1)}, N_{T}^{(2)}\right)$ in Theorem 3.2. The joint Laplace transform of the process $\left(\lambda_{T}^{(1)}, \lambda_{T}^{(2)}\right)$ can be also derived using this theorem as presented in Jang and Dassios (2013).

3.1. Joint Laplace Transform - Probability Generating Function of $\left(\lambda_{t}^{(1)}, \lambda_{t}^{(2)}\right.$, $\left.N_{t}^{(1)}, N_{t}^{(2)}, L_{t}^{(1)}, L_{t}^{(2)}\right)$ 
Theorem 3.1 Considering the constants, $0 \leq \theta \leq 1,0 \leq \eta \leq 1, \nu \geq 0, \zeta \geq 0$, $v \geq 0, \gamma \geq 0$ and time $0 \leq t \leq T$, we have the conditional joint Laplace transform, probability generating function of the process $\left(\lambda_{T}^{(1)}, \lambda_{T}^{(2)}\right)$, the point process $\left(N_{T}^{(1)}, N_{T}^{(2)}\right)$ and the compound point process $\left(L_{T}^{(1)}, L_{T}^{(2)}\right)$ is given by

$$
\begin{aligned}
& \left.E\left[\theta^{\left\{N_{T}^{(1)}-N_{t}^{(1)}\right.}\right\}_{\eta}\left\{N_{T}^{(2)}-N_{t}^{(2)}\right\} e^{-\nu\left\{L_{T}^{(1)}-L_{t}^{(1)}\right\}} e^{-\zeta\left\{L_{T}^{(2)}-L_{t}^{(2)}\right\}} \times e^{-v \lambda_{T}^{(1)}} e^{-\gamma \lambda_{T}^{(2)}} \mid \Im_{t}\right] \\
& =e^{-B_{1}(t) \lambda_{t}^{(1)}} e^{-B_{2}(t) \lambda_{t}^{(2)}} e^{-\{C(T)-C(t)\}},
\end{aligned}
$$

where $B_{1}(t)$ and $B_{2}(t)$ are determined by two non-linear ordinary differential equations $(O D E s)$

$$
\begin{aligned}
& -B_{1}^{\prime}(t)+\delta^{(1)} B_{1}(t)+\theta \hat{g}\left\{B_{1}(t)\right\} \hat{j}(\nu)-1=0 \\
& -B_{2}^{\prime}(t)+\delta^{(2)} B_{2}(t)+\eta \hat{h}\left\{B_{2}(t)\right\} \hat{k}(\zeta)-1=0
\end{aligned}
$$

with the boundary condition $B_{1}(T)=v$ and $B_{2}(T)=\gamma$, respectively, where

$$
\begin{aligned}
\hat{g}(\varepsilon) & =\int_{0}^{\infty} e^{-\varepsilon y} d G(y), \hat{h}(\varepsilon)=\int_{0}^{\infty} e^{-\varepsilon z} d H(z), \hat{j}(\kappa)=\int_{0}^{\infty} e^{-\kappa \zeta^{(1)}} d J\left(\zeta^{(1)}\right) \\
\text { and } \hat{k}(\kappa) & =\int_{0}^{\infty} e^{-\kappa \zeta^{(2)}} d K\left(\zeta^{(2)}\right) .
\end{aligned}
$$

$C(t)$ is determined by

$$
C(t)=\rho \int_{0}^{t}\left[1-\hat{f}\left\{B_{1}(s), B_{2}(s)\right\}\right] d s+a^{(1)} \delta^{(1)} \int_{0}^{t} B_{1}(s) d s+a^{(2)} \delta^{(2)} \int_{0}^{t} B_{2}(s) d s
$$

where

$$
\hat{f}(\varepsilon, \kappa)=\int_{0}^{\infty} \int_{0}^{\infty} e^{-\varepsilon x^{(1)}} e^{-\kappa x^{(2)}} d F\left(x^{(1)}, x^{(2)}\right)
$$

It is assumed that the Laplace transforms of above, i.e. $\stackrel{\wedge}{g}(\varepsilon), \hat{h}(\varepsilon), \hat{j}(\kappa), \hat{k}(\kappa)$ and the joint Laplace transform, $\hat{f}(\varepsilon, \kappa)$ are finite.

Proof. Consider a function $f\left(\lambda^{(1)}, n^{(1)}, l^{(1)}, \lambda^{(2)}, n^{(2)}, l^{(2)}, t\right)$ with an exponential affine form

$$
\begin{aligned}
& f\left(\lambda^{(1)}, n^{(1)}, l^{(1)}, \lambda^{(2)}, n^{(2)}, l^{(2)}, t\right) \\
= & \theta^{n^{(1)}} \eta^{n^{(2)}} e^{-\nu l^{(1)}} e^{-\zeta l^{(2)}} e^{-B_{1}(t) \lambda^{(1)}} e^{-B_{2}(t) \lambda^{(2)}} e^{C(t)},
\end{aligned}
$$


substitute into $\mathcal{A} f=0$ in (2.3), we have

$$
\begin{aligned}
& -\lambda^{(1)} B_{1}^{\prime}(t)-\lambda^{(2)} B_{2}^{\prime}(t)+C^{\prime}(t) \\
& +\lambda^{(1)}\left[\theta \hat{g}\left\{B_{1}(t)\right\} \hat{j}(\nu)\right]+\lambda^{(2)}\left[\eta \hat{h}\left\{B_{2}(t)\right\} \hat{k}(\zeta)\right] \\
& +\delta^{(1)}\left(a^{(1)}-\lambda^{(1)}\right)\left\{-B_{1}(t)\right\}+\delta^{(2)}\left(a^{(2)}-\lambda^{(2)}\right)\left\{-B_{2}(t)\right\} \\
& +\rho\left[\hat{f}\left\{B_{1}(t), B_{2}(t)\right\}-1\right] \\
& =0 \text {. } \\
& {\left[-B_{1}^{\prime}(t)+\delta^{(1)} B_{1}(t)+\theta \hat{g}\left\{B_{1}(t)\right\} \hat{j}(\nu)-1\right] \lambda^{(1)}} \\
& {\left[-B_{2}^{\prime}(t)+\delta^{(2)} B_{2}(t)+\eta \hat{h}\left\{B_{2}(t)\right\} \hat{k}(\zeta)-1\right] \lambda^{(2)}} \\
& +\left[C^{\prime}(t)+\rho \hat{f}\left\{B_{1}(t), B_{2}(t)\right\}-\rho-\delta^{(1)} a^{(1)} B_{1}(t)-\delta^{(2)} a^{(2)} B_{2}(t)\right] \\
& =0 \text {. }
\end{aligned}
$$

where

$$
\begin{aligned}
\hat{g}(\varepsilon) \hat{j}(\kappa) & =\int_{0}^{\infty} \int_{0}^{\infty} e^{-\varepsilon y} e^{-\kappa \zeta^{(1)}} d G(y) d J\left(\zeta^{(1)}\right), \\
\hat{h}(\varepsilon) \hat{k}(\kappa) & =\int_{0}^{\infty} \int_{0}^{\infty} e^{-\varepsilon z} e^{-\kappa \zeta^{(2)}} d H(z) d K\left(\zeta^{(2)}\right), \\
\hat{f}(\varepsilon, \kappa) & =\int_{0}^{\infty} \int_{0}^{\infty} e^{-\varepsilon x^{(1)}} e^{-\kappa x^{(2)}} d F\left(x^{(1)}, x^{(2)}\right) .
\end{aligned}
$$

Since this equation holds for any $l^{(1)}, l^{(2)}, n^{(1)}, n^{(2)}, \lambda^{(1)}$ and $\lambda^{(2)}$, it is equivalent to solving three separated equations, i.e.

$$
\begin{aligned}
&-B_{1}^{\prime}(t)+\delta^{(1)} B_{1}(t)+\theta \hat{g}\left\{B_{1}(t)\right\} \hat{j}(\nu)-1=0 \\
&-B_{2}^{\prime}(t)+\delta^{(2)} B_{2}(t)+\eta \hat{h}\left\{B_{2}(t)\right\} \hat{k}(\zeta)-1=0 \\
& C^{\prime}(t)+\rho \hat{f}\left\{B_{1}(t), B_{2}(t)\right\}-\rho-\delta^{(1)} a^{(1)} B_{1}(t)-\delta^{(2)} a^{(2)} B_{2}(t)=0
\end{aligned}
$$

We have two ODEs of (3.8.1) and (3.8.2) with the boundary condition $B_{1}(T)=v$ and $B_{2}(T)=\gamma$, respectively. By (3.8.3) with boundary condition $C(0)=0$, the integration of 
(3.5) follows. Since $\theta^{N_{t}^{(1)}} \eta^{N_{t}^{(2)}} e^{-\nu L_{t}^{(1)}} e^{-\zeta L_{t}^{(2)}} e^{-B_{1}(t) \lambda_{t}^{(1)}} e^{-B_{2}(t) \lambda_{t}^{(2)}} e^{C(t)}$ is a $\Im$-martingale by the property of the infinitesimal generator, we have

$$
\begin{aligned}
& E\left[\theta^{N_{T}^{(1)}} \eta^{N_{T}^{(2)}} e^{-\nu L_{T}^{(1)}} e^{-\zeta L_{T}^{(2)}} e^{-B_{1}(T) \lambda_{T}^{(1)}} e^{-B_{1}(T) \lambda_{T}^{(2)}} e^{C(T)} \mid \Im_{t}\right] \\
= & \theta^{N_{t}^{(1)}} \eta^{N_{t}^{(2)}} e^{-\nu L_{t}^{(1)}} e^{-\zeta L_{t}^{(2)}} e^{-B_{1}(t) \lambda_{t}^{(1)}} e^{-B_{2}(t) \lambda_{t}^{(2)}} e^{C(t)} .
\end{aligned}
$$

Then, by the boundary condition $B_{1}(T)=v$ and $B_{2}(T)=\gamma,(3.1)$ follows.

\subsection{Joint Laplace Transform of $\left(\lambda_{T}^{(1)}, \lambda_{T}^{(2)}\right)$}

Based on (3.1), we can easily derive the joint Laplace transform for the process $\left(\lambda_{T}^{(1)}\right.$, $\left.\lambda_{T}^{(2)}\right)$ setting $\theta=1, \eta=1, \nu=0, \zeta=0$. As it has already presented in Jang and Dassios (2013), we state two propositions adopted from them in this section. $\mathcal{G}_{v, 1}^{-1}(T)$ and $\mathcal{H}_{\gamma, 1}^{-1}(T)$ in the proposition will become apparent in Theorem 3.3.

Proposition 3.1. The conditional joint Laplace transform for the process $\left(\lambda_{T}^{(1)}, \lambda_{T}^{(2)}\right)$ given $\lambda_{0}^{(1)}$ and $\lambda_{0}^{(2)}$ at time $t=0$ is given by

$$
\begin{aligned}
& E\left[e^{-v \lambda_{T}^{(1)}} e^{-\gamma \lambda_{T}^{(2)}} \mid \lambda_{0}^{(1)}, \lambda_{0}^{(2)}\right] \exp \left\{-\mathcal{G}_{v, 1}^{-1}(T) \lambda_{0}^{(1)}\right\} \exp \left\{-\mathcal{H}_{\gamma, 1}^{-1}(T) \lambda_{0}^{(2)}\right\} \\
& \times \exp \left[-\rho \int_{0}^{T}\left[1-\hat{f}\left\{\mathcal{G}_{v, 1}^{-1}(\tau), \mathcal{H}_{\gamma, 1}^{-1}(\tau)\right\}\right] d \tau\right] \\
& \times \exp \left[-\int_{0}^{v}\left\{\frac{a^{(1)} \delta^{(1)} u}{\delta^{(1)} u+\hat{g}(u)-1}\right\} d u\right] \\
& \times \exp \left[-\int_{\mathcal{H}_{\gamma, 1}^{-1}(T)}^{\gamma}\left\{\frac{a^{(2)} \delta^{(2)} u}{\delta^{(2)} u+\hat{h}(u)-1}\right\} d u\right]
\end{aligned}
$$

where

$$
\begin{gathered}
\mu_{1_{G}}=\int_{0}^{\infty} y d G(y), \quad \mathcal{G}_{v, 1}\left(\Psi_{1}\right)=: \int_{\Psi_{1}}^{v}\left[\frac{1}{\delta^{(1)} u+\hat{g}(u)-1}\right] d u \\
\mu_{1_{H}}=\int_{0}^{\infty} z d H(z), \quad \mathcal{H}_{\gamma, 1}\left(\Psi_{2}\right)=: \int_{\Psi_{2}}^{\gamma}\left[\frac{1}{\delta^{(2)} u+\hat{h}(u)-1}\right] d u \\
\delta^{(1)}>\mu_{1_{G}} \text { and } \delta^{(2)}>\mu_{1_{H}} .
\end{gathered}
$$


Remark 1. (3.10) is the conditional joint Laplace transform of the process $\left(\lambda_{T}^{(1)}, \lambda_{T}^{(2)}\right)$ given $\lambda_{0}^{(1)}$ and $\lambda_{0}^{(2)}$ at time $t=0$, where the jumps $X^{(1)}$ and $X^{(2)}$ with distribution function $F\left(x^{(1)}, x^{(2)}\right)$, occur simultaneously/collaterally with constant intensity $\rho$. Because of these two dependences in the process, this conditional joint Laplace transform is not the product of conditional Laplace transform of $\lambda_{T}^{(1)}$ given $\lambda_{0}^{(1)}$ and the Laplace transform of $\lambda_{T}^{(2)}$ given $\lambda_{0}^{(2)}$, i.e.

$$
E\left[e^{-v \lambda_{T}^{(1)}} e^{-\gamma \lambda_{T}^{(2)}} \mid \lambda_{0}^{(1)}, \lambda_{0}^{(2)}\right] \neq E\left[e^{-v \lambda_{T}^{(1)}} \mid \lambda_{0}^{(1)}\right] E\left[e^{-\gamma \lambda_{T}^{(2)}} \mid \lambda_{0}^{(2)}\right]
$$

Proposition 3.2. The joint Laplace transform of the asymptotic distribution of $\left(\lambda_{T}^{(1)}, \lambda_{T}^{(2)}\right)$ is given by

$$
\begin{aligned}
& \lim _{T \rightarrow \infty} E\left[e^{-v \lambda_{T}^{(1)}} e^{-\gamma \lambda_{T}^{(2)}} \mid \lambda_{0}^{(1)}, \lambda_{0}^{(2)}\right]= \exp \left[-\rho \int_{0}^{\infty}\left[1-\hat{f}\left\{\mathcal{G}_{v, 1}^{-1}(\tau), \mathcal{H}_{\gamma, 1}^{-1}(\tau)\right\}\right] d \tau\right] \\
& \times \exp \left[-\int_{0}^{v}\left\{\frac{a^{(1)} \delta^{(1)} u}{\delta^{(1)} u+\hat{g}(u)-1}\right\} d u\right] \\
& \times \exp \left[-\int_{0}^{\gamma}\left\{\frac{a^{(2)} \delta^{(2)} u}{\delta^{(2)} u+\hat{h}(u)-1}\right\} d u\right]
\end{aligned}
$$

where $\delta^{(1)}>\mu_{1_{G}}$ and $\delta^{(2)}>\mu_{1_{H}}$.

Remark 2. We can easily derive the Laplace transform of $\lambda_{T}^{(1)}$ and $\lambda_{T}^{(2)}$ for a fixed time $T$, respectively using (3.10). This can also be found in Theorem 3.2 in Dassios and Zhao (2011). Setting $\rho=0$, we can obtain the conditional Laplace transform of $\lambda_{T}^{(d)}(d=1,2)$ given $\lambda_{0}^{(d)}$ at time $t=0$ for the self-exciting process with exponential decay. These processes can be considered in modelling the bivariate intensity process only when self-excited jumps are involved eliminating the effect of the externally excited jumps, or to see the contribution of "after-cyber attacks" to the intensity eliminating the contribution of "initial-cyber attacks" to the intensity in cyber insurance context.

\subsection{Joint Probability Generating Function of $\left(N_{T}^{(1)}, N_{T}^{(2)}\right)$}

We derive the joint probability generating function for the process $\left(N_{T}^{(1)}, N_{T}^{(2)}\right)$ for a fixed time $T$ in Theorem 3.2 using the result in Theorem 3.1.

Theorem 3.2. The conditional joint probability generating function for the process $\left(N_{T}^{(1)}, N_{T}^{(2)}\right)$ given $\lambda_{0}^{(1)}$ and $\lambda_{0}^{(2)}$, and $N_{0}^{(1)}=0$ and $N_{0}^{(2)}=0$ at time $t=0$ is given by 


$$
\begin{aligned}
& E\left[\theta^{N_{T}^{(1)}} \eta^{N_{T}^{(2)}} \mid \lambda_{0}^{(1)}, \lambda_{0}^{(2)}\right] \\
= & \exp \left\{-\mathcal{G}_{0, \theta}^{-1}(T) \lambda_{0}^{(1)}\right\} \exp \left\{-\mathcal{H}_{0, \eta}^{-1}(T) \lambda_{0}^{(2)}\right\} \\
\times & \exp \left[-\rho \int_{0}^{T}\left[1-\hat{f}\left\{\mathcal{G}_{0, \theta}^{-1}(\tau), \mathcal{H}_{0, \eta}^{-1}(\tau)\right\}\right] d \tau\right] \\
\times & \exp \left[-\int_{0}^{\mathcal{G}_{0, \theta}^{-1}(T)}\left\{\frac{a^{(1)} \delta^{(1)} u}{1-\delta^{(1)} u-\theta \hat{g}(u)}\right\} d u\right] \\
\times & \exp \left[-\int_{0}^{\mathcal{H}_{0, \eta}^{-1}(T)}\left\{\frac{a^{(2)} \delta^{(2)} u}{1-\delta^{(2)} u-\eta \hat{h}(u)}\right\} d u\right] .
\end{aligned}
$$

Proof. By setting $t=0, \nu=0, \zeta=0, v=0$ and $\gamma=0$ in (3.1) with the assumption that $N_{0}^{(1)}=0$ and $N_{0}^{(2)}=0$, we have

$$
E\left[\theta^{N_{T}^{(1)}} \eta^{N_{T}^{(2)}} \mid \Im_{0}\right]=e^{-B_{1}(0) \lambda_{0}^{(1)}} e^{-B_{2}(0) \lambda_{0}^{(2)}} e^{-C(T)},
$$

where $B_{1}(0)$ is uniquely determined by the non-linear ordinary differential equation (ODE)

$$
-B_{1}^{\prime}(t)+\delta^{(1)} B_{1}(t)+\theta \hat{g}\left\{B_{1}(t)\right\}-1=0
$$

with boundary condition $B_{1}(T)=0$ and similarly, $B_{2}(0)$ is uniquely determined by the non-linear ODE

$$
-B_{2}^{\prime}(t)+\delta^{(2)} B_{2}(t)+\eta \hat{h}\left\{B_{2}(t)\right\}-1=0
$$

with boundary condition $B_{2}(T)=0$.

(3.15) can be solved, under the condition $\delta^{(1)}>\mu_{1_{G}}$, by the following steps (1)-(7).

(1) Set $B_{1}(t)=\Psi_{1}(T-t)=\Psi_{1}(\tau)$. Then it becomes

$$
\frac{d \Psi_{1}(\tau)}{d \tau}=1-\delta^{(1)} B_{1}(t)-\theta \hat{g}\left\{B_{1}(t)\right\}=1-\delta_{1}^{(1)} \Psi_{1}(\tau)-\theta \hat{g}\left\{\Psi_{1}(\tau)\right\}=: f_{1}\left(\Psi_{1}\right), \quad 0 \leq \theta \leq 1
$$

with initial condition $\Psi_{1}(0)=0$; we define the right-hand side as the function, $f_{1}\left(\Psi_{1}\right)$.

(2) There is only one positive singular point, denoted by $v^{*}>0$, which can be obtained by solving the equation

$$
1-\delta^{(1)} u-\theta \hat{g}(u)=0,
$$

at which the uniqueness of the solution of equation (3.18) is violated. This is because, for the case $0<\theta<1, f_{1}\left(\Psi_{1}\right)=0$ is equivalent to

$$
\hat{g}(u)=\frac{1}{\theta}\left(1-\delta^{(1)} u\right), \quad 0<\theta<1 .
$$


Note that the left-hand side of (3.19) is a convex function, hence it is clear that there is only one positive solution to $f_{1}\left(\Psi_{1}\right)$. For the case that $\theta=0$, there is only one singular point

$$
v^{*}=\frac{1}{\delta^{(1)}}>0
$$

For both cases, we have

$$
v^{*}=\frac{1-\theta \hat{g}\left(v^{*}\right)}{\delta^{(1)}} \geq \frac{1-\theta}{\delta^{(1)}}>0,
$$

hence, we have $f_{1}\left(\Psi_{1}\right)>0$ for $0 \leq \Psi_{1}<v^{*}$ and $f_{1}\left(\Psi_{1}\right)<0$ for $\Psi_{1}>v^{*}$.

(3) (3.17) can be written as

$$
\frac{d \Psi_{1}(\tau)}{1-\delta_{1}^{(1)} \Psi_{1}(\tau)-\theta \hat{g}\left\{\Psi_{1}(\tau)\right\}}=d \tau .
$$

Integrate both sides from time 0 to $\tau$, then we have

$$
\int_{0}^{\Psi_{1}(\tau)}\left[\frac{1}{1-\delta^{(1)} u-\theta \hat{g}(u)}\right] d u=\tau
$$

where $0 \leq \Psi_{1}(\tau)<v^{*}$. Now we define the left-hand side as the function

$$
\mathcal{G}_{0, \theta}\left(\Psi_{1}\right)=: \int_{0}^{\Psi_{1}(\tau)}\left[\frac{1}{1-\delta^{(1)} u-\theta \hat{g}(u)}\right] d u .
$$

Then we have

$$
\mathcal{G}_{0, \theta}\left(\Psi_{1}\right)=\tau(=T-t),
$$

which is the time difference between $T$ and $t$, and it is obvious that $\Psi_{1}(\tau) \rightarrow 0$ when $\tau \rightarrow 0$ and $\Psi_{1}(\tau) \rightarrow v^{*}$ when $\tau \rightarrow \infty$. The integrand is positive in the domain $u \in\left(0, v^{*}\right]$ and for $\Psi_{1}(\tau) \geq 0, \mathcal{G}_{0, \theta}\left(\Psi_{1}\right)$ is a strictly increasing function. Therefore

$$
\mathcal{G}_{0, \theta}\left(\Psi_{1}\right)=\tau:\left[0, v^{*}\right) \rightarrow[0, \infty)
$$

is a well defined function and it inverse function

$$
\mathcal{G}_{0, \theta}^{-1}(\tau)=\Psi_{1}:[0, \infty) \rightarrow\left[0, v^{*}\right)
$$

exists.

(4) The unique solution is found by

$$
\Psi_{1}(\tau)=\Psi_{1}(T-t)=B_{1}(t)=\mathcal{G}_{0, \theta}^{-1}(\tau)=\mathcal{G}_{0, \theta}^{-1}(T-t)
$$

and hence $B_{1}(0)$ is obtained,

$$
B_{1}(0)=\Psi_{1}(T)=\mathcal{G}_{0, \theta}^{-1}(T)
$$


(5) Similar to solving (3.15), under the condition $\delta^{(2)}>\mu_{1_{H}}$, the unique solution for (3.16) is given by

$$
\Psi_{2}(\tau)=\Psi_{2}(T-t)=B_{2}(t)=\mathcal{H}_{0, \eta}^{-1}(\tau)=\mathcal{H}_{0, \eta}^{-1}(T-t)
$$

and hence $B_{2}(0)$ is obtained,

$$
B_{2}(0)=\Psi_{2}(T)=\mathcal{H}_{0, \eta}^{-1}(T)
$$

where

$$
\mathcal{H}_{0, \eta}\left(\Psi_{2}\right)=\int_{0}^{\Psi_{2}(\tau)}\left[\frac{1}{1-\delta^{(2)} u-\eta \hat{h}(u)}\right] d u
$$

is also a strictly increasing function: the integrand is positive in the domain $u \in\left(0, \gamma^{*}\right]$ and for $\Psi_{2}(\tau) \geq 0$ and

$$
\mathcal{H}_{0, \eta}\left(\Psi_{2}\right)=\tau:\left[0, \gamma^{*}\right) \rightarrow[0, \infty)
$$

is a well defined function and it inverse function

$$
\mathcal{H}_{0, \eta}^{-1}(\tau)=\Psi_{2}:[0, \infty) \rightarrow\left[0, \gamma^{*}\right)
$$

exists.

(6) $C(T)$ is determined by

$$
C(T)=\rho \int_{0}^{T}\left[1-\hat{f}\left\{\mathcal{G}_{0, \theta}^{-1}(\tau), \mathcal{H}_{0, \eta}^{-1}(\tau)\right\}\right] d \tau+\delta^{(1)} a^{(1)} \int_{0}^{T} \mathcal{G}_{0, \theta}^{-1}(\tau) d \tau+\delta^{(2)} a^{(2)} \int_{0}^{T} \mathcal{H}_{0, \eta}^{-1}(\tau) d \tau
$$

and by the change of variable $\mathcal{G}_{0, \theta}^{-1}(\tau)=u$, we have $\tau=\mathcal{G}_{0, \theta}(u)\left(\rightarrow d \tau=\frac{\partial \mathcal{G}_{0, \theta}(u)}{\partial u} d u\right)$, and

$$
\int_{0}^{T} \mathcal{G}_{0, \theta}^{-1}(\tau) d \tau=\int_{0}^{\mathcal{G}_{0, \theta}^{-1}(T)} \frac{u}{1-\delta^{(1)} u-\theta \hat{g}(u)} d u
$$

and similarly, $\mathcal{H}_{0, \eta}^{-1}(\tau)=u$, we have $\tau=\mathcal{H}_{0, \eta}(u)\left(\rightarrow d \tau=\frac{\partial \mathcal{H}_{0, \eta}(u)}{\partial u} d u\right)$, and

$$
\int_{0}^{T} \mathcal{H}_{0, \eta}^{-1}(\tau) d \tau=\int_{0}^{\mathcal{H}_{0, \eta}^{-1}(T)} \frac{u}{1-\delta^{(2)} u-\eta \hat{h}(u)} d u
$$

(7) Finally, substitute $B_{1}(0), B_{2}(0)$ and $C(T)$ into (3.14) and the result follows.

Remark 3. We can easily derive the Laplace transform of $N_{T}^{(1)}$ and $N_{T}^{(2)}$ for a fixed time $T$, respectively, using (3.13). This can also be found in Theorem 3.4 in Dassios and Zhao (2011). Setting $\rho=0$, we can obtain the conditional Laplace transform of $N_{T}^{(d)}(d=1,2)$ given $\lambda_{0}^{(d)}$ at time $t=0$ for the self-exciting process with exponential decay. These processes can be considered in modelling the bivariate point process only when self-excited jumps are 
involved in the bivariate intensity process eliminating the effect of the externally excited jumps, or to see the number of losses from the contribution of "after-cyber attacks" to the intensity eliminating the contribution of "initial-cyber attacks" to the intensity in cyber insurance context.

\subsection{Joint Laplace Transform of $\left(L_{T}^{(1)}, L_{T}^{(2)}\right)$}

To derive the joint Laplace transform of the process $\left(L_{T}^{(1)}, L_{T}^{(2)}\right)$ for a fixed time $T$, we start with deriving the conditional joint Laplace transform, probability generating function of the process $\left(\lambda_{T}^{(1)}, \lambda_{T}^{(2)}\right)$ and the compound point process $\left(L_{T}^{(1)}, L_{T}^{(2)}\right)$ in Theorem 3.3.

Theorem 3.3 The conditional joint Laplace transform, probability generating function of the process $\left(\lambda_{T}^{(1)}, \lambda_{T}^{(2)}\right)$ and the compound point process $\left(L_{T}^{(1)}, L_{T}^{(2)}\right)$ given $\lambda_{0}^{(1)}$ and $\lambda_{0}^{(2)}$, and $L_{0}^{(1)}=0$ and $L_{0}^{(2)}=0$ at time $t=0$ is given by

$$
\begin{aligned}
E\left[e^{-\nu L_{T}^{(1)}} e^{-\zeta L_{T}^{(2)}} \times e^{-v \lambda_{T}^{(1)}} e^{-\gamma \lambda_{T}^{(2)}} \mid \lambda_{0}^{(1)}, \lambda_{0}^{(2)}\right] \\
=\exp \left\{-\mathcal{G}_{v, \nu}^{-1}(T) \lambda_{0}^{(1)}\right\} \exp \left\{-\mathcal{H}_{\gamma, \zeta}^{-1}(T) \lambda_{0}^{(2)}\right\} \\
\times \exp \left[-\rho \int_{0}^{T}\left[1-\hat{f}\left\{\mathcal{G}_{v, \nu}^{-1}(\tau), \mathcal{H}_{\gamma, \zeta}^{-1}(\tau)\right\}\right] d \tau\right] \\
\times \exp \left[-\int_{\mathcal{G}_{v, \nu}^{-1}(T)}^{v}\left\{\frac{a^{(1)} \delta^{(1)} u}{\delta^{(1)} u+\hat{j}(\nu) \hat{g}(u)-1}\right\} d u\right] \\
\times \exp \left[-\int_{\mathcal{H}_{\gamma, \zeta}^{-1}(T)}^{\gamma}\left\{\frac{a^{(2)} \delta^{(2)} u}{\delta^{(2)} u+\hat{k}(\xi) \hat{h}(u)-1}\right\} d u\right]
\end{aligned}
$$

where

$$
\begin{gathered}
\mu_{1_{G}}=\int_{0}^{\infty} y d G(y), \quad \mathcal{G}_{v, \nu}\left(\Psi_{1}\right)=\int_{\Psi_{1}}^{v}\left[\frac{1}{\delta^{(1)} u+\hat{j}(\nu) \hat{g}(u)-1}\right] d u \\
\mu_{1_{H}}=\int_{0}^{\infty} z d H(z), \quad \mathcal{H}_{\gamma, \zeta}\left(\Psi_{2}\right)=\int_{\Psi_{2}}^{\gamma}\left[\frac{1}{\delta^{(2)} u+\hat{k}(\zeta) \hat{h}(u)-1}\right] d u \\
\delta^{(1)}>\hat{j}(\nu) \mu_{1_{G}} \text { and } \delta^{(2)}>\hat{k}(\xi) \mu_{1_{H}} .
\end{gathered}
$$

Proof. By setting $t=0, \theta=1$, and $\eta=1$, in (3.1), we have

$$
E\left[e^{-\nu L_{T}^{(1)}} e^{-\zeta L_{T}^{(2)}} e^{-v \lambda_{T}^{(1)}} e^{-\gamma \lambda_{T}^{(2)}} \mid \Im_{0}\right]=e^{-B_{1}(0) \lambda_{0}^{(1)}} e^{-B_{2}(0) \lambda_{0}^{(2)}} e^{-C(T)},
$$


where $B_{1}(0)$ is uniquely determined by the non-linear ordinary differential equation (ODE)

$$
-B_{1}^{\prime}(t)+\delta^{(1)} B_{1}(t)+\stackrel{\wedge}{g}\left\{B_{1}(t)\right\} \hat{j}(\nu)-1=0
$$

with boundary condition $B_{1}(T)=v$, and similarly $B_{2}(0)$ is uniquely determined by the non-linear ODE

$$
-B_{2}^{\prime}(t)+\delta^{(2)} B_{2}(t)+\hat{h}\left\{B_{2}(t)\right\} \hat{k}(\zeta)-1=0
$$

with boundary condition $B_{2}(T)=\gamma$.

(3.22) can be solved, under the condition $\delta^{(1)}>\hat{j}(\nu) \mu_{1_{G}}$, by the following steps $(1)-(8)$ :

(1) Let us set $B_{1}(t)=\Psi_{1}(T-t)=\Psi_{1}(\tau)$. Then it becomes

$$
\frac{d \Psi_{1}(\tau)}{d \tau}=1-\delta^{(1)} B_{1}(t)-\hat{g}\left\{B_{1}(t)\right\} \hat{j}(\nu)=1-\delta^{(1)} \Psi_{1}(\tau)-\hat{g}\left\{\Psi_{1}(\tau)\right\} \hat{j}(\nu)=: f_{2}\left(\Psi_{1}\right)
$$

with initial condition $\Psi_{1}(0)=v$; we define the right-hand side as the function, $f_{2}\left(\Psi_{1}\right)$.

(2) For $\nu=0$, we have

$$
f_{2}\left(\Psi_{1}\right)=1-\delta^{(1)} \Psi_{1}(\tau)-\hat{g}\left\{\Psi_{1}(\tau)\right\}
$$

and its unique solution is found by $\Psi_{1}(\tau)=\mathcal{G}_{v, 1}^{-1}(\tau)$, that has been shown in Proposition 3.1.

Under the condition of $\delta^{(1)}>\hat{j}(\nu) \mu_{1_{G}}$, we have

$\frac{\partial f_{2}\left(\Psi_{1}\right)}{\partial \Psi_{1}}=\hat{j}(\nu) \int_{0}^{\infty} y e^{-\Psi_{1} y} d G(y)-\delta^{(1)} \leq \hat{j}(\nu) \int_{0}^{\infty} y d G(y)-\delta^{(1)}=\hat{j}(\nu) \mu_{1_{G}}-\delta^{(1)}<0$, for $\Psi_{1} \geq 0$ then $f_{2}\left(\Psi_{1}\right)<0$ for $\Psi_{1}>0$.

(3) (3.24) can be written as

$$
\frac{d \Psi_{1}(\tau)}{\delta^{(1)} \Psi_{1}(\tau)-\hat{j}(\nu) \hat{g}\left\{\Psi_{1}(\tau)\right\}-1}=-d \tau
$$

Integrate both sides from time 0 to $\tau$ with initial condition $\Psi_{1}(0)=v>0$, then we have

$$
\int_{\Psi_{1}}^{v}\left[\frac{1}{\delta^{(1)} u+\hat{j}(\nu) \hat{g}(u)-1}\right] d u=\tau
$$

where $\Psi_{1} \geq 0$. Now we define the left-hand side as the function

$$
\mathcal{G}_{v, \nu}\left(\Psi_{1}\right)=: \int_{\Psi_{1}}^{v}\left[\frac{1}{\delta^{(1)} u+\hat{j}(\nu) \hat{g}(u)-1}\right] d u
$$


Then we have

$$
\mathcal{G}_{v, \nu}\left(\Psi_{1}\right)=\tau(=T-t),
$$

which is the time difference between $T$ and $t$ and it is obvious that $\Psi_{1} \rightarrow v$ when $\tau(=$ $T-t) \rightarrow 0$.

(4) As $\delta^{(1)}-\hat{j}(\nu) \mu_{1_{G}}>0$ by convergence test, we have

$$
\int_{0}^{v}\left[\frac{1}{\delta^{(1)} u+\hat{j}(\nu) \hat{g}(u)-1}\right] d u=\infty
$$

so $\Psi_{1} \rightarrow 0$ when $\tau \rightarrow \infty$. The integrand is positive in the domain $u \in(0, v]$ and for $\Psi_{1} \leq v, \mathcal{G}_{v, \nu}\left(\Psi_{1}\right)$ is a strictly decreasing function. Therefore

$$
\mathcal{G}_{v, \nu}\left(\Psi_{1}\right)=\tau:(0, v] \rightarrow[0, \infty)
$$

is a well defined (monotone) function and its inverse function

$$
\mathcal{G}_{v, \nu}^{-1}(\tau)=\Psi_{1}:[0, \infty) \rightarrow(0, v]
$$

exists.

(5) The unique solution is found by

$$
\Psi_{1}(\tau)=\Psi_{1}(T-t)=B_{1}(t)=\mathcal{G}_{v, \nu}^{-1}(\tau)=\mathcal{G}_{v, \nu}^{-1}(T-t)
$$

and hence $B_{1}(0)$ is obtained,

$$
B_{1}(0)=\Psi_{1}(T)=\mathcal{G}_{v, \nu}^{-1}(T) .
$$

(6) Similar to solving (3.22), under the condition $\delta^{(2)}>\hat{k}(\zeta) \mu_{1_{H}}$, the unique solution for (3.23) is found by

$$
\Psi_{2}(\tau)=\Psi_{2}(T-t)=B_{2}(t)=\mathcal{H}_{\gamma, \zeta}^{-1}(\tau)=\mathcal{H}_{\gamma, \zeta}^{-1}(T-t)
$$

and hence $B_{2}(0)$ is obtained,

$$
B_{2}(0)=\Psi_{2}(T)=\mathcal{H}_{\gamma, \zeta}^{-1}(T)
$$

Hence

$$
\mathcal{H}_{\gamma, \zeta}\left(\Psi_{2}\right)=: \int_{\Psi_{2}}^{\gamma}\left[\frac{1}{\delta^{(2)} u+\hat{k}(\zeta) \hat{h}(u)-1}\right] d u
$$

is a strictly decreasing function, where the integrand is positive in the domain $u \in(0, \gamma]$ and for $\Psi_{2} \leq \gamma, \mathcal{H}_{\gamma, \zeta}\left(\Psi_{2}\right)$ is a strictly decreasing function. Therefore

$$
\mathcal{H}_{\gamma, \zeta}\left(\Psi_{2}\right)=\tau:(0, \gamma] \rightarrow[0, \infty)
$$


is a well defined (monotone) function and its inverse function

$$
\mathcal{H}_{\gamma, \zeta}^{-1}(\tau)=\Psi_{2}:[0, \infty) \rightarrow(0, \gamma]
$$

exists.

(7) Now $C(T)$ is determined by

$$
C(T)=\rho \int_{0}^{T}\left[1-\hat{f}\left\{\mathcal{G}_{v, \nu}^{-1}(\tau), \mathcal{H}_{\gamma, \zeta}^{-1}(\tau)\right\}\right] d \tau+a^{(1)} \delta^{(1)} \int_{0}^{T} \mathcal{G}_{v, \nu}^{-1}(\tau) d \tau+a^{(2)} \delta^{(2)} \int_{0}^{T} \mathcal{H}_{\gamma, \zeta}^{-1}(\tau) d \tau
$$

By the change of variable $\mathcal{G}_{v, \nu}^{-1}(\tau)=u$, we have $\tau=\mathcal{G}_{v, \nu}^{-1}(u)$, and

$$
\int_{0}^{T} \mathcal{G}_{v, \nu}^{-1}(\tau) d \tau=\int_{\mathcal{G}_{v, \nu}^{-1}(0)}^{\mathcal{G}_{v, \nu}^{-1}(T)} u \frac{\partial \tau}{\partial u} d u=\int_{\mathcal{G}_{v, \nu}^{-1}(T)}^{v}\left\{\frac{u}{\delta^{(1)} u+\hat{j}(\nu) \hat{g}(u)-1}\right\} d u
$$

Similarly, we have

$$
\int_{0}^{T} \mathcal{H}_{\gamma, \zeta}^{-1}(\tau) d \tau=\int_{\mathcal{H}_{\gamma, \zeta}^{-1}(0)}^{\mathcal{H}_{\gamma, \zeta}^{-1}(T)} u \frac{\partial \tau}{\partial u} d u=\int_{\mathcal{H}_{\gamma, \zeta}^{-1}(\tau)(T)}^{\gamma}\left\{\frac{u}{\delta^{(2)} u+\hat{k}(\xi) \hat{h}(u)-1}\right\} d u
$$

(8) Finally, substitute $B_{1}(0), B_{2}(0)$ and $C(T)$ into (3.21) and the result follows.

Now let us derive the joint Laplace transform of the process $\left(L_{T}^{(1)}, L_{T}^{(2)}\right)$ for a fixed time $T$ in Theorem 3.4.

Theorem 3.4. The conditional joint Laplace transform of the process $\left(L_{T}^{(1)}, L_{T}^{(2)}\right)$ given $\lambda_{0}^{(1)}$ and $\lambda_{0}^{(2)}$, and $L_{0}^{(1)}=0$ and $L_{0}^{(2)}=0$ at time $t=0$ is given by

$$
\begin{aligned}
& E\left[e^{-\nu L_{T}^{(1)}} e^{-\zeta L_{T}^{(2)}} \mid \lambda_{0}^{(1)}, \lambda_{0}^{(2)}\right] \exp \left\{-\mathcal{G}_{0, \nu}^{-1}(T) \lambda_{0}^{(1)}\right\} \exp \left\{-\mathcal{H}_{0, \zeta}^{-1}(T) \lambda_{0}^{(2)}\right\} \\
& \times \exp \left[-\rho \int\left[1-\hat{f}\left\{\mathcal{G}_{0, \nu}^{-1}(\tau), \mathcal{H}_{0, \zeta}^{-1}(\tau)\right\}\right] d \tau\right] \\
& \times \exp \left[-\int_{0}^{T}\left\{\frac{a^{(1)} \delta^{(1)} u}{\delta^{(1)} u+\hat{j}(\nu) \hat{g}(u)-1}\right\} d u\right] \\
& \times \exp \left[-\int_{\mathcal{H}_{0, \zeta}^{-1}(T)}^{0}\left\{\frac{a^{(2)} \delta^{(2)} u}{\delta^{(2)} u+\hat{k}(\xi) \hat{h}(u)-1}\right\} d u\right] .
\end{aligned}
$$


Proof. Set $v=0$, and $\gamma=0$ in (3.20), then the result follows immediately.

Remark 4. We can easily derive the Laplace transform of $L_{T}^{(1)}$ and $L_{T}^{(2)}$ for a fixed time $T$, respectively, using (3.25). Setting $\rho=0$, we can obtain the conditional Laplace transform of $L_{T}^{(d)}(d=1,2)$ given $\lambda_{0}^{(d)}$ at time $t=0$ for the self-exciting process with exponential decay. These processes can be considered in modelling the bivariate compound point process only when self-excited jumps are involved in the bivariate intensity process eliminating the effect of the externally excited jumps, or to see the aggregate losses from the contribution of "aftercyber attacks" to the intensity eliminating the contribution of "initial-cyber attacks" to the intensity in cyber insurance context.

\section{Moments, covariance and linear correlation}

In this section, we derive the expectation of $L_{t}^{(i)}(i=1,2)$ and the joint expectation of $L_{t}^{(1)}$ and $L_{t}^{(2)}$, which is another key result of this paper, for which we need the expectations of $\lambda_{t}^{(1)}$ and $\lambda_{t}^{(2)}$, respectively and the joint expectation of $\lambda_{t}^{(1)}$ and $\lambda_{t}^{(2)}$. So let us start with stating three propositions adopted from Dassios and Zhao (2011) and Jang and Dassios (2013).

Proposition 4.1. The conditional expectation of the process $\lambda_{t}^{(1)}$ given $\lambda_{0}^{(1)}$ at time $t=0$, is given by

$$
\begin{aligned}
& E\left(\lambda_{t}^{(1)} \mid \lambda_{0}^{(1)}\right)=\lambda_{0}^{(1)} e^{-\left(\delta^{(1)}-\mu_{1_{G}}\right) t}+\frac{\mu_{1_{F_{1}}} \rho+a^{(1)} \delta^{(1)}}{\delta^{(1)}-\mu_{1_{G}}}\left(1-e^{-\left(\delta^{(1)}-\mu_{1_{G}}\right) t}\right), \text { for } \delta^{(1)} \neq \mu_{1_{G}}, \\
& E\left(\lambda_{t}^{(1)} \mid \lambda_{0}^{(1)}\right)=\lambda_{0}^{(1)}+\left(\mu_{1_{F_{1}}} \rho+a^{(1)} \delta^{(1)}\right) t, \text { for } \delta^{(1)}=\mu_{1_{G}},
\end{aligned}
$$

where

$$
\mu_{1_{F_{1}}}=\int_{0}^{\infty} x^{(1)} d F\left(x^{(1)}\right)
$$

and $F\left(x^{(1)}\right)$ is the marginal distribution function for $\left\{X_{i}^{(1)}\right\}_{i=1,2, \cdots}$.

The conditional expectation of the process $\lambda_{t}^{(2)}$ given $\lambda_{0}^{(2)}$ at time $t=0$, is given by

$$
\begin{aligned}
& E\left(\lambda_{t}^{(2)} \mid \lambda_{0}^{(2)}\right)=\lambda_{0}^{(2)} e^{-\left(\delta^{(2)}-\mu_{1_{H}}\right) t}+\frac{\mu_{1_{F_{2}}} \rho+a^{(2)} \delta^{(2)}}{\delta^{(2)}-\mu_{1_{H}}}\left(1-e^{-\left(\delta^{(2)}-\mu_{1_{H}}\right) t}\right), \text { for } \delta^{(2)} \neq \mu_{1_{H}}, \\
& E\left(\lambda_{t}^{(2)} \mid \lambda_{0}^{(2)}\right)=\lambda_{0}^{(2)}+\left(\mu_{1_{F_{2}}} \rho+a^{(2)} \delta^{(2)}\right) t, \text { for } \delta^{(2)}=\mu_{1_{H}},
\end{aligned}
$$

where

$$
\mu_{1_{F_{2}}}=\int_{0}^{\infty} x^{(2)} d F\left(x^{(2)}\right)
$$


and $F\left(x^{(2)}\right)$ is the marginal distribution function for $\left\{X_{i}^{(2)}\right\}_{i=1,2, \cdots}$.

Assuming that $\delta^{(1)}>\mu_{1_{G}}$ and $\delta^{(2)}>\mu_{1_{H}}$, and setting time $t \rightarrow \infty$ in (4.1) and (4.3) respectively, the expectations of the stationary distribution of the process $\lambda_{t}^{(i)}(i=1,2)$ are given by

$$
E\left(\lambda_{t}^{(1)}\right)=\frac{\mu_{1_{F_{1}}} \rho+a^{(1)} \delta^{(1)}}{\delta^{(1)}-\mu_{1_{G}}}
$$

and

$$
E\left(\lambda_{t}^{(2)}\right)=\frac{\mu_{1_{F_{2}}} \rho+a^{(2)} \delta^{(2)}}{\delta^{(2)}-\mu_{1_{H}}} .
$$

Proposition 4.2. The conditional joint expectation of $\lambda_{t}^{(1)}$ and $\lambda_{t}^{(2)}$ given $\lambda_{0}^{(1)}$ and $\lambda_{0}^{(2)}$ at time $t=0$, is given by

$$
\begin{aligned}
& E\left(\lambda_{t}^{(1)} \lambda_{t}^{(2)} \mid \lambda_{0}^{(1)}, \lambda_{0}^{(2)}\right)=\lambda_{0}^{(1)} \lambda_{0}^{(2)} e^{-\left\{\left(\delta^{(1)}-\mu_{1_{G}}\right)+\left(\delta^{(2)}-\mu_{1_{H}}\right)\right\} t} \\
& +\left(a^{(2)} \delta^{(2)}+\mu_{1_{F_{2}}} \rho\right)\left[\begin{array}{c}
\left(\lambda_{0}^{(1)}-\frac{\mu_{1_{F_{1}}} \rho+a^{(1)} \delta^{(1)}}{\delta^{(1)}-\mu_{1_{G}}}\right)\left\{\frac{e^{-\left(\delta^{(1)}-\mu_{1_{G}}\right) t}-e^{-\left\{\left(\delta^{(1)}-\mu_{1_{G}}\right)+\left(\delta^{(2)}-\mu_{1}{ }_{H}\right)\right\}_{t}}}{\delta^{(2)}-\mu_{1_{H}}}\right\} \\
+\left(\frac{\mu_{1_{1}} \rho+a^{(1)} \delta^{(1)}}{\delta^{(1)}-\mu_{1_{G}}}\right)\left\{\frac{1-e^{-\left\{\left(\delta^{(1)}-\mu_{1_{G}}\right)+\left(\delta^{(2)}-\mu_{1_{H}}\right)\right\} t}}{\left(\delta^{(1)}-\mu_{1_{G}}\right)+\left(\delta^{(2)}-\mu_{1_{H}}\right)}\right\}
\end{array}\right] \\
& +\left(a^{(1)} \delta^{(1)}+\mu_{1_{F_{1}}} \rho\right)\left[\begin{array}{c}
\left(\lambda_{0}^{(2)}-\frac{\mu_{1_{F_{2}}} \rho+a^{(2)} \delta^{(2)}}{\delta^{(2)}-\mu_{1_{H}}}\right)\left\{\frac{e^{-\left(\delta^{(2)}-\mu_{1}\right) t}-e^{-\left\{\left(\delta^{(1)}-\mu_{1}\right)+\left(\delta^{(2)}-\mu_{1}\right)\right\} t}}{\delta^{(1)}-\mu_{1_{G}}}\right\} \\
+\left(\frac{\mu_{F_{2}} \rho+a^{(2)} \delta^{(2)}}{\delta^{(2)}-\mu_{1_{H}}}\right)\left\{\frac{1-e^{-\left\{\left(\delta^{(1)}-\mu_{1_{G}}\right)+\left(\delta^{(2)}-\mu_{1_{H}}\right)\right\} t}}{\left(\delta^{(1)}-\mu_{1_{G}}\right)+\left(\delta^{(2)}-\mu_{1_{H}}\right)}\right\}
\end{array}\right] \\
& +\mu_{1_{F_{1,2}}} \rho\left\{\frac{1-e^{-\left\{\left(\delta^{(1)}-\mu_{1_{G}}\right)+\left(\delta^{(2)}-\mu_{1_{H}}\right)\right\} t}}{\left(\delta^{(1)}-\mu_{1_{G}}\right)+\left(\delta^{(2)}-\mu_{1_{H}}\right)}\right\}, \quad \text { for } \delta^{(1)} \neq \mu_{1_{G}} \text { and } \delta^{(2)} \neq \mu_{1_{H}} \text {. } \\
& E\left(\lambda_{t}^{(1)} \lambda_{t}^{(2)} \mid \lambda_{0}^{(1)}, \lambda_{0}^{(2)}\right)=\lambda_{0}^{(1)} \lambda_{0}^{(2)} \\
& +\left(a^{(2)} \delta^{(2)}+\mu_{1_{F_{2}}} \rho\right)\left[\lambda_{0}^{(1)} t+\left(\frac{\mu_{1_{F_{1}}} \rho+a^{(1)} \delta^{(1)}}{2}\right) t^{2}\right] \\
& +\left(a^{(1)} \delta^{(1)}+\mu_{1_{F_{1}}} \rho\right)\left[\lambda_{0}^{(2)} t+\left(\frac{\mu_{1_{F_{2}}} \rho+a^{(2)} \delta^{(2)}}{2}\right) t^{2}\right]
\end{aligned}
$$




$$
+\mu_{1_{F_{1,2}}} \rho t, \quad \text { for } \delta^{(1)}=\mu_{1_{G}} \quad \text { and } \quad \delta^{(2)}=\mu_{1_{H}} .
$$

where $\mu_{1_{F_{1,2}}}=\int_{0}^{\infty} \int_{0}^{\infty} x^{(1)} x^{(2)} d F\left(x^{(1)}, x^{(2)}\right)$.

Assuming that $\delta^{(1)}>\mu_{1_{G}}$ and $\delta^{(2)}>\mu_{1_{H}}$, and setting time $t \rightarrow \infty$ in (4.7), the joint expectation of the stationary distribution of the process $\lambda_{t}^{(i)}(i=1,2)$ is given by

$$
E\left(\lambda_{t}^{(1)} \lambda_{t}^{(2)}\right)=\left(\frac{1}{\left(\delta^{(1)}-\mu_{1_{G}}\right)+\left(\delta^{(2)}-\mu_{1_{H}}\right)}\right) \times\left\{\begin{array}{c}
\left(a^{(2)} \delta^{(2)}+\mu_{1_{F_{2}}} \rho\right)\left(\frac{\mu_{1_{F_{F}}} \rho+a^{(1)} \delta^{(1)}}{\delta^{(1)}-\mu_{1_{G}}}\right) \\
+\left(a^{(1)} \delta^{(1)}+\mu_{1_{F_{1}}} \rho\right)\left(\frac{\mu_{1_{F_{2}}} \rho+a^{(2)} \delta^{(2)}}{\delta^{(2)}-\mu_{1_{H}}}\right) \\
+\mu_{1_{F_{1,2}} \rho} \rho
\end{array}\right\}
$$

Proposition 4.3. The second moment of the process $\lambda_{t}^{(1)}$ given $\lambda_{0}^{(1)}$ at time $t=0$, is given by

$$
\begin{gathered}
E\left[\left\{\lambda_{t}^{(1)}\right\}^{2} \mid \lambda_{0}^{(1)}\right] \\
=\left(\lambda_{0}^{(1)}\right)^{2} e^{-2\left(\delta^{(1)}-\mu_{1_{G}}\right) t}+\frac{2\left(\mu_{1_{F_{1}}} \rho+a^{(1)} \delta^{(1)}\right)+\mu_{2_{G}}}{\delta^{(1)}-\mu_{1_{G}}} \\
\quad \times\left(\lambda_{0}^{(1)}-\frac{\mu_{1_{F_{1}}} \rho+a^{(1)} \delta^{(1)}}{\delta^{(1)}-\mu_{1_{G}}}\right)\left(e^{-\left(\delta^{(1)}-\mu_{1_{G}}\right) t}-e^{-2\left(\delta^{(1)}-\mu_{1_{G}}\right) t}\right) \\
+\left[\frac{\left\{2\left(\mu_{1_{F_{1}}} \rho+a^{(1)} \delta^{(1)}\right)+\mu_{2_{G}}\right\}\left(\mu_{1_{F_{1}}} \rho+a^{(1)} \delta^{(1)}\right)}{2\left(\delta^{(1)}-\mu_{1_{G}}\right)^{2}}+\frac{\mu_{2_{F_{1}}} \rho}{2\left(\delta^{(1)}-\mu_{1_{G}}\right)}\right]\left(1-e^{-2\left(\delta^{(1)}-\mu_{1_{G}}\right) t}\right), \\
\quad \text { for } \delta^{(1)} \neq \mu_{1_{G}}, \\
E\left[\left\{\lambda_{t}^{(1)}\right\}^{2} \mid \lambda_{0}^{(1)}\right] \quad \text { for } \delta^{(1)}=\mu_{1_{G}},
\end{gathered}
$$


where

$$
\mu_{1_{F_{1}}}=\int_{0}^{\infty} x^{(1)} d F\left(x^{(1)}\right), \quad \text { and } \mu_{2_{F_{1}}}=\int_{0}^{\infty}\left\{x^{(1)}\right\}^{2} d F\left(x^{(1)}\right)
$$

and $F\left(x^{(1)}\right)$ is the marginal distribution function for $\left\{X_{i}^{(1)}\right\}_{i=1,2, \cdots}$.

The second moment of the process $\lambda_{t}^{(2)}$ given $\lambda_{0}^{(2)}$ at time $t=0$, is given by

$$
\begin{aligned}
& E\left[\left\{\lambda_{t}^{(2)}\right\}^{2} \mid \lambda_{0}^{(2)}\right] \\
& =\left(\lambda_{0}^{(2)}\right)^{2} e^{-2\left(\delta^{(2)}-\mu_{1_{H}}\right) t}+\frac{2\left(\mu_{1_{F_{2}}} \rho+a^{(2)} \delta^{(2)}\right)+\mu_{2_{H}}}{\delta^{(2)}-\mu_{1_{H}}} \\
& \times\left(\lambda_{0}^{(2)}-\frac{\mu_{1_{F_{2}}} \rho+a^{(2)} \delta^{(2)}}{\delta^{(2)}-\mu_{1_{H}}}\right)\left(e^{-\left(\delta^{(2)}-\mu_{1_{H}}\right) t}-e^{-2\left(\delta^{(2)}-\mu_{1_{H}}\right) t}\right) \\
& +\left[\frac{\left\{2\left(\mu_{1_{F_{2}}} \rho+a^{(2)} \delta^{(2)}\right)+\mu_{2_{H}}\right\}\left(\mu_{1_{F_{2}}} \rho+a^{(2)} \delta^{(2)}\right)}{2\left(\delta^{(2)}-\mu_{1_{H}}\right)^{2}}+\frac{\mu_{2_{F_{2}}} \rho}{2\left(\delta^{(2)}-\mu_{1_{H}}\right)}\right]\left(1-e^{-2\left(\delta^{(2)}-\mu_{1_{H}}\right) t}\right), \\
& \text { for } \delta^{(2)} \neq \mu_{1_{H}} \\
& E\left[\left\{\lambda_{t}^{(2)}\right\}^{2} \mid \lambda_{0}^{(2)}\right] \\
& =\left(\lambda_{0}^{(2)}\right)^{2}+\left\{2\left(\mu_{1_{F_{2}}} \rho+a^{(2)} \delta^{(2)}\right)+\mu_{2_{H}}\right\}\left\{\lambda_{0}^{(2)} t+\frac{1}{2}\left(\mu_{1_{F_{2}}} \rho+a^{(2)} \delta^{(2)}\right) t^{2}\right\}+\mu_{2_{F_{2}}} \rho t \\
& \text { for } \delta^{(2)}=\mu_{1_{H}} \text {, }
\end{aligned}
$$

where

$$
\mu_{1_{F_{2}}}=\int_{0}^{\infty} x^{(2)} d F\left(x^{(2)}\right) \text { and } \mu_{2_{F_{2}}}=\int_{0}^{\infty}\left\{x^{(2)}\right\}^{2} d F\left(x^{(2)}\right)
$$

and $F\left(x^{(2)}\right)$ is the marginal distribution function for $\left\{X_{i}^{(2)}\right\}_{i=1,2, \cdots}$.

Assuming that $\delta^{(1)}>\mu_{1_{G}}$ and $\delta^{(2)}>\mu_{1_{H}}$, and setting time $t \rightarrow \infty$ in (4.10) and (4.12) respectively, the second moments of the stationary distribution of the process $\lambda_{t}^{(i)}(i=1,2)$ are given by 


$$
E\left[\left\{\lambda_{t}^{(1)}\right\}^{2}\right]=\frac{\left\{2\left(\mu_{1_{F_{1}}} \rho+a^{(1)} \delta^{(1)}\right)+\mu_{2_{G}}\right\}\left(\mu_{1_{F_{1}}} \rho+a^{(1)} \delta^{(1)}\right)}{2\left(\delta^{(1)}-\mu_{1_{G}}\right)^{2}}+\frac{\mu_{2_{F_{1}}} \rho}{2\left(\delta^{(1)}-\mu_{1_{G}}\right)}
$$

and

$$
E\left[\left\{\lambda_{t}^{(2)}\right\}^{2}\right]=\frac{\left\{2\left(\mu_{1_{F_{2}}} \rho+a^{(2)} \delta^{(2)}\right)+\mu_{2_{H}}\right\}\left(\mu_{1_{F_{2}}} \rho+a^{(2)} \delta^{(2)}\right)}{2\left(\delta^{(2)}-\mu_{1_{H}}\right)^{2}}+\frac{\mu_{2_{F_{2}}} \rho}{2\left(\delta^{(2)}-\mu_{1_{H}}\right)} .
$$

Using Proposition 4.1, we now derive the expectation of $L_{T}^{(i)}(i=1,2)$ directly solving an ODE in Theorem 4.1. We can derive them by differentiating the Laplace transform of $L_{T}^{(i)}(i=1,2)$ with respect to $\nu$ and $\xi$, and then setting $\nu=0$ and $\xi=0$, respectively. However solving the ODE directly is easier to generalise to derive higher moments beyond the conditions $\delta^{(1)}>\mu_{1_{G}}$ and $\delta^{(2)}>\mu_{1_{H}}$, if necessary.

The moments of $N_{t}$ can also be derived directly solving relevant ODEs, for which we refer you Dassios and Zhao $(2011,2017)$.

Theorem 4.1. The conditional expectation of the process $L_{t}^{(1)}$ given $\lambda_{0}^{(1)}$ at time $t=0$, is given by

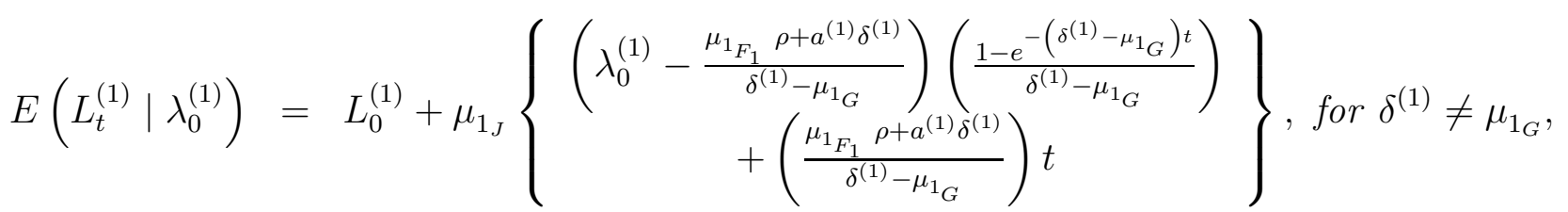

$E\left(L_{t}^{(1)} \mid \lambda_{0}^{(1)}\right)=L_{0}^{(1)}+\mu_{1_{J}}\left\{\lambda_{0}^{(1)} t+\left(\frac{\mu_{1_{F_{1}}} \rho+a^{(1)} \delta^{(1)}}{2}\right) t^{2}\right\}$, for $\delta^{(1)}=\mu_{1_{G}}$,

where

$$
\mu_{1_{J}}=\int_{0}^{\infty} \zeta^{(1)} d J\left(\zeta^{(1)}\right)
$$

The conditional expectation of the process $L_{t}^{(2)}$ given $\lambda_{0}^{(2)}$ at time $t=0$, is given by

$$
\begin{aligned}
& E\left(L_{t}^{(2)} \mid \lambda_{0}^{(2)}\right)=L_{0}^{(2)}+\mu_{1_{K}}\left\{\begin{array}{c}
\left(\lambda_{0}^{(2)}-\frac{\mu_{1_{F_{2}}} \rho+a^{(2)} \delta^{(2)}}{\delta^{(2)}-\mu_{1_{H}}}\right)\left(\frac{1-e^{-\left(\delta^{(2)}-\mu_{1}\right)^{t}}}{\delta^{(2)}-\mu_{1_{H}}}\right) \\
+\left(\frac{\mu_{1_{2}} \rho+a^{(2)} \delta^{(2)}}{\delta^{(2)}-\mu_{1_{H}}}\right) t
\end{array}\right\}, \text { for } \delta^{(2)} \neq \mu_{1_{H}}, \\
& E\left(L_{t}^{(2)} \mid \lambda_{0}^{(2)}\right)=L_{0}^{(2)}+\mu_{1_{K}}\left\{\lambda_{0}^{(2)} t+\left(\frac{\mu_{1_{F_{2}}} \rho+a^{(2)} \delta^{(2)}}{2}\right) t^{2}\right\}, \text { for } \delta^{(2)}=\mu_{1_{H}},
\end{aligned}
$$


where

$$
\mu_{1_{K}}=\int_{0}^{\infty} \zeta^{(2)} d K\left(\zeta^{(2)}\right)
$$

Proof. See Appendix A.

Corollary 4.1. For the stationary distribution of the process $\lambda_{t}^{(1)}$, given $L_{0}^{(1)}=0$, the expectation of the process $L_{t}^{(1)}$ is given by

$$
E\left(L_{t}^{(1)}\right)=\mu_{1_{J}}\left(\frac{\mu_{1_{F_{1}}} \rho+a^{(1)} \delta^{(1)}}{\delta^{(1)}-\mu_{1_{G}}}\right) t, \quad \delta^{(1)}>\mu_{1_{G}}
$$

and for the stationary distribution of the process $\lambda_{t}^{(2)}$, given $L_{0}^{(2)}=0$, the expectation of the process $L_{t}^{(2)}$ is given by

$$
E\left(L_{t}^{(2)}\right)=\mu_{1_{K}}\left(\frac{\mu_{1_{F_{2}}} \rho+a^{(2)} \delta^{(2)}}{\delta^{(2)}-\mu_{1_{H}}}\right) t, \quad \delta^{(2)}>\mu_{1_{H}} .
$$

Proof. See Appendix B.

We now derive the joint expectation of $L_{t}^{(1)}$ and $L_{t}^{(2)}$ in Theorem 3.6, for which we start with a lemma to show the joint expectation of $\lambda_{t}^{(1)} L_{t}^{(2)}$ and the joint expectation of $\lambda_{t}^{(2)} L_{t}^{(1)}$, respectively. For simplicity, we use the case for the stationary distribution of the process $\lambda_{t}^{(i)}(i=1,2)$. It can serve a reasonable approximation for the joint expectation of $L_{t}^{(1)}$ and $L_{t}^{(2)}$ provided that the process the has been running for a relatively long period and is close to the stationary (asymptotic) state.

Lemma 4.1. For the stationary distribution of the process $\lambda_{t}^{(1)}$ and $\lambda_{t}^{(2)}$, given $L_{0}^{(2)}=0$, the joint expectation of $\lambda_{t}^{(1)}$ and $L_{t}^{(2)}$ is given by

$$
\left.\begin{array}{c}
E\left(\lambda_{t}^{(1)} L_{t}^{(2)}\right)=\mu_{1_{K}}\left(a^{(1)} \delta^{(1)}+\mu_{1_{F_{1}}} \rho\right)\left\{\frac{\mu_{1_{F_{2}}} \rho+a^{(2)} \delta^{(2)}}{\left(\delta^{(2)}-\mu_{1_{H}}\right)\left(\delta^{(1)}-\mu_{1_{G}}\right)}\right\} t \\
+\mu_{1_{K}}\left(\frac{1-e^{-\left(\delta^{(1)}-\mu_{1_{G}}\right) t}}{\delta^{(1)}-\mu_{1_{G}}}\right) \\
\times\left[\left(\frac{1}{\left.\left(\frac{1}{\left(\delta^{(1)}-\mu_{1_{G}}\right)+\left(\delta^{(2)}-\mu_{1_{H}}\right)}\right)\left\{+\left(a^{(1)} \delta^{(1)}+\mu_{\left.1_{F_{1}} \rho\right)\left(\frac{\mu_{1_{F}} \rho+a^{(2)} \delta^{(2)}}{\delta^{(2)}-\mu_{1_{H}}}\right)}\right)+\mu_{1_{F_{1,2}}} \rho\right\}\right]}\right]\right\} \\
-\left(a^{(1)} \delta^{(1)}+\mu_{1_{F_{1}}} \rho\right)\left\{\frac{\mu_{1_{F_{2}}} \rho+a^{(2)} \delta^{(2)}}{\left(\delta^{(2)}-\mu_{1_{H}}\right)\left(\delta^{(1)}-\mu_{1_{G}}\right)}\right\}
\end{array}\right]
$$

and given $L_{0}^{(1)}=0$, the joint expectation of $\lambda_{t}^{(2)}$ and $L_{t}^{(1)}$ is given by 


$$
\begin{aligned}
& E\left(\lambda_{t}^{(2)} L_{t}^{(1)}\right)=\mu_{1_{J}}\left(a^{(2)} \delta^{(2)}+\mu_{1_{F_{2}}} \rho\right)\left\{\frac{\mu_{1_{F_{1}}} \rho+a^{(1)} \delta^{(1)}}{\left(\delta^{(2)}-\mu_{1_{H}}\right)\left(\delta^{(1)}-\mu_{1_{G}}\right)}\right\} t \\
& +\mu_{1_{J}}\left(\frac{1-e^{-\left(\delta^{(2)}-\mu_{1_{H}}\right) t}}{\delta^{(2)}-\mu_{1_{H}}}\right)
\end{aligned}
$$

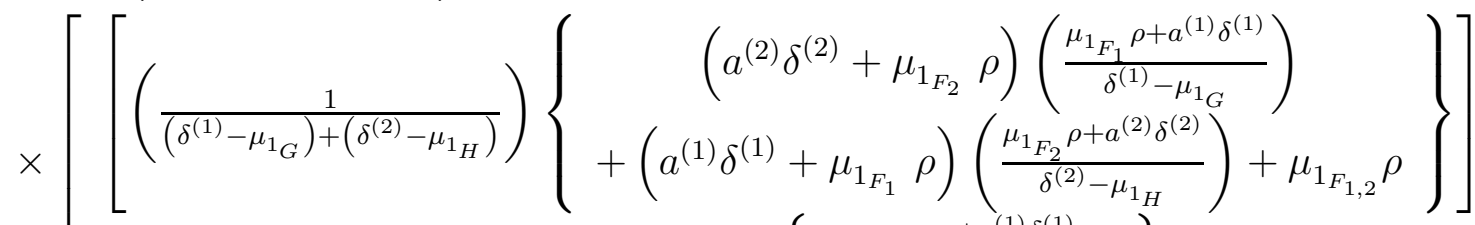

$$
\begin{aligned}
& -\left(a^{(2)} \delta^{(2)}+\mu_{1_{F_{2}}} \rho\right)\left\{\frac{\mu_{1_{F_{1}}} \rho+a^{(1)} \delta^{(1)}}{\left(\delta^{(2)}-\mu_{1_{H}}\right)\left(\delta^{(1)}-\mu_{1_{G}}\right)}\right\} \\
& \text { for } \delta^{(1)}>\mu_{1_{G}} \text { and } \delta^{(2)}>\mu_{1_{H}},
\end{aligned}
$$

Proof. See Appendix C.

Theorem 4.2. For the stationary distribution of the process $\lambda_{t}^{(1)}$ and $\lambda_{t}^{(2)}$, given $L_{0}^{(1)}=$ $L_{0}^{(2)}=0$, the joint expectation of $L_{t}^{(1)}$ and $L_{t}^{(2)}$ is given by

$$
\begin{aligned}
& E\left(L_{t}^{(1)} L_{t}^{(2)}\right)=\mu_{1_{J}} \mu_{1_{K}}\left(\frac{a^{(1)} \delta^{(1)}+\mu_{1_{F_{1}}} \rho}{2}\right)\left\{\frac{\mu_{1_{F_{2}}} \rho+a^{(2)} \delta^{(2)}}{\left(\delta^{(2)}-\mu_{1_{H}}\right)\left(\delta^{(1)}-\mu_{1_{G}}\right)}\right\} t^{2} \\
& +\mu_{1_{J}} \mu_{1_{K}}\left(\frac{1}{\delta^{(1)}-\mu_{1_{G}}}\right)\left\{t-\left(\frac{1-e^{-\left(\delta^{(1)}-\mu_{1_{G}}\right) t}}{\delta^{(1)}-\mu_{1_{G}}}\right)\right\} \\
& \times\left[\begin{array}{c}
{\left[\left(\frac{1}{\left(\delta^{(1)}-\mu_{1_{G}}\right)+\left(\delta^{(2)}-\mu_{1_{H}}\right)}\right)\left\{\begin{array}{c}
\left(a^{(2)} \delta^{(2)}+\mu_{1_{F_{2}}} \rho\right)\left(\frac{\mu_{1_{1}} \rho+a^{(1)} \delta^{(1)}}{\delta^{(1)}-\mu_{1_{G}}}\right) \\
+\left(a^{(1)} \delta^{(1)}+\mu_{1_{F_{1}}} \rho\right)\left(\frac{\mu_{1_{F_{2}}} \rho+a^{(2)} \delta^{(2)}}{\delta^{(2)}-\mu_{1_{H}}}\right)+\mu_{1_{F_{1,2}} \rho} \rho
\end{array}\right\}\right]} \\
-\left(a^{(1)} \delta^{(1)}+\mu_{1_{F_{1}}} \rho\right)\left\{\frac{\mu_{1_{F_{2}}} \rho+a^{(2)} \delta^{(2)}}{\left(\delta^{(2)}-\mu_{1_{H}}\right)\left(\delta^{(1)}-\mu_{1_{G}}\right)}\right\}
\end{array}\right] \\
& +\mu_{1_{K}} \mu_{1_{J}}\left(\frac{a^{(2)} \delta^{(2)}+\mu_{1_{F_{2}}} \rho}{2}\right)\left\{\frac{\mu_{1_{F_{1}}} \rho+a^{(1)} \delta^{(1)}}{\left(\delta^{(2)}-\mu_{1_{H}}\right)\left(\delta^{(1)}-\mu_{1_{G}}\right)}\right\} t^{2} \\
& +\mu_{1_{K}} \mu_{1_{J}}\left(\frac{1}{\delta^{(2)}-\mu_{1_{H}}}\right)\left\{t-\left(\frac{1-e^{-\left(\delta^{(2)}-\mu_{1_{H}}\right) t}}{\delta^{(2)}-\mu_{1_{H}}}\right)\right\} \\
& \times\left[\begin{array}{c}
{\left[\left(\frac{1}{\left(\delta^{(1)}-\mu_{1_{G}}\right)+\left(\delta^{(2)}-\mu_{1_{H}}\right)}\right)\left\{\begin{array}{c}
\left(a^{(2)} \delta^{(2)}+\mu_{1_{F_{2}}} \rho\right)\left(\frac{\mu_{1_{F_{1}}} \rho+a^{(1)} \delta^{(1)}}{\delta^{(1)}-\mu_{1_{G}}}\right) \\
+\left(a^{(1)} \delta^{(1)}+\mu_{1_{F_{1}}} \rho\right)\left(\frac{\mu_{1_{F_{2}}} \rho+a^{(2)} \delta^{(2)}}{\delta^{(2)}-\mu_{1_{H}}}\right)+\mu_{1_{F_{1,2}} \rho} \rho
\end{array}\right\}\right]} \\
-\left(a^{(2)} \delta^{(2)}+\mu_{1_{F_{2}}} \rho\right)\left\{\frac{\mu_{1_{F_{1}}} \rho+a^{(1)} \delta^{(1)}}{\left(\delta^{(2)}-\mu_{1_{H}}\right)\left(\delta^{(1)}-\mu_{1_{G}}\right)}\right\}
\end{array}\right],
\end{aligned}
$$




$$
\text { for } \delta^{(1)} \neq \mu_{1_{G}} \text { and } \delta^{(2)} \neq \mu_{1_{H}} \text {. }
$$

Proof. See Appendix D.

Based on Theorem 4.2 and Corollary 4.1, we can easily obtain the covariance between $L_{t}^{(1)}$ and $L_{t}^{(2)}$, i.e.

$$
\operatorname{Cov}\left(L_{t}^{(1)}, L_{t}^{(2)}\right)=E\left(L_{t}^{(1)} L_{t}^{(2)}\right)-E\left(L_{t}^{(1)}\right) E\left(L_{t}^{(2)}\right)
$$

and the linear correlation coefficient between $L_{t}^{(1)}$ and $L_{t}^{(2)}$, i.e.

$$
\operatorname{Corr}\left(L_{t}^{(1)}, L_{t}^{(2)}\right)=\frac{\operatorname{Cov}\left(L_{t}^{(1)}, L_{t}^{(2)}\right)}{\sqrt{\operatorname{Var}\left(L_{t}^{(1)}\right)} \sqrt{\operatorname{Var}\left(L_{t}^{(2)}\right)}}
$$

and hence we omit their corresponding expressions. We show their numerical values in cyber insurance context in Section 5 .

For the correlation coefficient calculation, we need variance of $L_{t}^{(1)}$ and $L_{t}^{(2)}$, respectively, for which we start with a lemma to show the joint expectation of $\lambda_{t}^{(1)} L_{t}^{(1)}$ and the joint expectation of $\lambda_{t}^{(2)} L_{t}^{(2)}$, respectively.

Lemma 4.2. For the stationary distribution of the process $\lambda_{t}^{(1)}$ and $\lambda_{t}^{(2)}$, given $L_{0}^{(1)}=0$, the joint expectation of $\lambda_{t}^{(1)}$ and $L_{t}^{(1)}$ is given by

$$
\begin{aligned}
& E\left(\lambda_{t}^{(1)} L_{t}^{(1)}\right)=\left(a^{(1)} \delta^{(1)}+\mu_{1_{F_{1}}} \rho\right) \mu_{1_{J}}\left\{\frac{\mu_{1_{F_{1}}} \rho+a^{(1)} \delta^{(1)}}{\left(\delta^{(1)}-\mu_{1_{G}}\right)^{2}}\right\}\left\{t-\left(\frac{1-e^{-\left(\delta^{(1)}-\mu_{1_{G}}\right) t}}{\delta^{(1)}-\mu_{1_{G}}}\right)\right\} \\
&+\mu_{1_{J}}\left(\frac{1-e^{-\left(\delta^{(1)}-\mu_{1_{G}}\right) t}}{\delta^{(1)}-\mu_{1_{G}}}\right) \\
& \times\left[\frac{\left\{2\left(\mu_{1_{F_{1}}} \rho+a^{(1)} \delta^{(1)}\right)+\mu_{2_{G}}\right\}\left(\mu_{1_{F_{1}}} \rho+a^{(1)} \delta^{(1)}\right)}{2\left(\delta^{(1)}-\mu_{1_{G}}\right)^{2}}+\frac{\mu_{2_{F_{1}}} \rho}{2\left(\delta^{(1)}-\mu_{1_{G}}\right)}\right] \\
&+\mu_{1_{G}} \mu_{1_{J}}\left(\frac{1-e^{-\left(\delta^{(1)}-\mu_{1_{G}}\right) t}}{\delta^{(1)}-\mu_{1_{G}}}\right)\left(\frac{\mu_{1_{F_{1}}} \rho+a^{(1)} \delta^{(1)}}{\delta^{(1)}-\mu_{1_{G}}}\right) \\
& \text { for } \delta^{(1)}>\mu_{1_{G}},
\end{aligned}
$$

and given $L_{0}^{(1)}=0$, the joint expectation of $\lambda_{t}^{(2)}$ and $L_{t}^{(2)}$ is given by

$$
E\left(\lambda_{t}^{(2)} L_{t}^{(2)}\right)=\left(a^{(2)} \delta^{(2)}+\mu_{1_{F_{2}}} \rho\right) \mu_{1_{K}}\left\{\frac{\mu_{1_{F_{2}}} \rho+a^{(2)} \delta^{(2)}}{\left(\delta^{(2)}-\mu_{1_{H}}\right)^{2}}\right\}\left\{t-\left(\frac{1-e^{-\left(\delta^{(2)}-\mu_{1_{H}}\right) t}}{\delta^{(2)}-\mu_{1_{H}}}\right)\right\}
$$




$$
\begin{aligned}
& +\mu_{1_{K}}\left(\frac{1-e^{-\left(\delta^{(2)}-\mu_{1_{H}}\right) t}}{\delta^{(2)}-\mu_{1_{H}}}\right) \\
& \times\left[\frac{\left\{2\left(\mu_{1_{F_{2}}} \rho+a^{(2)} \delta^{(2)}\right)+\mu_{2_{H}}\right\}\left(\mu_{1_{F_{2}}} \rho+a^{(2)} \delta^{(2)}\right)}{2\left(\delta^{(2)}-\mu_{1_{H}}\right)^{2}}+\frac{\mu_{2_{F_{2}}} \rho}{2\left(\delta^{(2)}-\mu_{1_{H}}\right)}\right] \\
& +\mu_{1_{H}} \mu_{1_{K}}\left(\frac{1-e^{-\left(\delta^{(2)}-\mu_{1_{H}}\right) t}}{\delta^{(2)}-\mu_{1_{H}}}\right)\left(\frac{\mu_{1_{F_{2}}} \rho+a^{(2)} \delta^{(2)}}{\delta^{(2)}-\mu_{1_{H}}}\right) \\
& \text { for } \delta^{(2)}>\mu_{1_{H}},
\end{aligned}
$$

Proof. See Appendix E.

Theorem 4.3. For the stationary distribution of the process $\lambda_{t}^{(1)}$ and $\lambda_{t}^{(2)}$, given $L_{0}^{(1)}=$ $L_{0}^{(2)}=0$, the second moment of the process of $L_{t}^{(1)}$ is given by

$$
\begin{aligned}
& E\left\{\left(L_{t}^{(1)}\right)^{2}\right\} \\
& {\left[\frac{a^{(1)} \delta^{(1)}+\mu_{1_{F_{1}}} \rho}{2}\right) \mu_{1_{J}}\left\{\frac{\mu_{1_{F_{1}}} \rho+a^{(1)} \delta^{(1)}}{\left(\delta^{(1)}-\mu_{1_{G}}\right)^{2}}\right\} t^{2}} \\
& -\left(a^{(1)} \delta^{(1)}+\mu_{1_{F_{1}}} \rho\right) \mu_{1_{J}}\left\{\frac{\mu_{1_{F_{1}}} \rho+a^{(1)} \delta^{(1)}}{\left(\delta^{(1)}-\mu_{1_{G}}\right)^{3}}\right\}\left\{t-\left(\frac{1-e^{-\left(\delta^{(1)}-\mu_{1_{G}}\right) t}}{\delta^{(1)}-\mu_{1_{G}}}\right)\right\}
\end{aligned}
$$

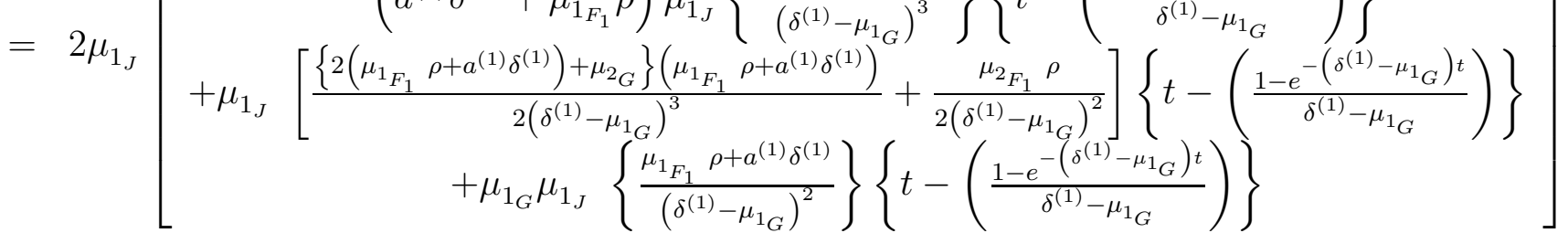

$$
\begin{aligned}
& +\mu_{2_{J}}\left(\frac{\mu_{1_{F_{1}}} \rho+a^{(1)} \delta^{(1)}}{\delta^{(1)}-\mu_{1_{G}}}\right) t
\end{aligned}
$$

and the second moment of the process of $L_{t}^{(2)}$ is given by

$$
E\left\{\left(L_{t}^{(2)}\right)^{2}\right\}
$$




$$
\begin{gathered}
=2 \mu_{1_{K}}\left[\begin{array}{c}
\left(\frac{a^{(2)} \delta^{(2)}+\mu_{1_{F_{2}}} \rho}{2}\right) \mu_{1_{K}}\left\{\frac{\mu_{1_{F_{2}}} \rho+a^{(2)} \delta^{(2)}}{\left.\delta^{(2)}-\mu_{1_{H}}\right)^{2}}\right\} t^{2} \\
-\left(a^{(2)} \delta^{(2)}+\mu_{1_{F_{2}}} \rho\right) \mu_{1_{K}}\left\{\frac{\mu_{1_{F_{2}}} \rho+a^{(2)} \delta^{(2)}}{\left(\delta^{(2)}-\mu_{1_{H}}\right)^{3}}\right\}\left\{t-\left(\frac{1-e^{-\left(\delta^{(2)}-\mu_{1}\right) t}}{\delta^{(2)}-\mu_{1_{H}}}\right)\right\} \\
+\mu_{1_{K}}\left[\frac{\left\{2\left(\mu_{1_{F_{2}}} \rho+a^{(2)} \delta^{(2)}\right)+\mu_{2_{H}}\right\}\left(\mu_{1_{F_{2}}} \rho+a^{(2)} \delta^{(2)}\right)}{2\left(\delta^{(2)}-\mu_{1_{H}}\right)^{3}}+\frac{\mu_{F_{2}} \rho}{2\left(\delta^{(2)}-\mu_{1_{H}}\right)^{2}}\right]\left\{t-\left(\frac{1-e^{-\left(\delta^{(2)}-\mu_{1_{H}}\right) t}}{\delta^{(2)}-\mu_{1_{H}}}\right)\right\} \\
+\mu_{1_{H}} \mu_{1_{K}}\left\{\frac{\mu_{1_{F_{2}}} \rho+a^{(2)} \delta^{(2)}}{\left(\delta^{(2)}-\mu_{1_{H}}\right)^{2}}\right\}\left\{t-\left(\frac{1-e^{-\left(\delta^{(2)}-\mu_{1_{H}}\right) t}}{\delta^{(2)}-\mu_{1_{H}}}\right)\right\}
\end{array}\right] \\
+\mu_{2_{K}}\left(\frac{\mu_{1_{F_{2}}} \rho+a^{(2)} \delta^{(2)}}{\delta^{(2)}-\mu_{1_{H}}}\right) t .
\end{gathered}
$$

Proof. See Appendix F.

Corollary 4.2. For the stationary distribution of the process $\lambda_{t}^{(1)}$ and $\lambda_{t}^{(2)}$, given $L_{0}^{(1)}=L_{0}^{(2)}=0$, the variance of the process of $L_{t}^{(1)}$ is given by

$$
\begin{gathered}
\operatorname{Var}\left(L_{t}^{(1)}\right) \\
=2 \mu_{1_{J}}\left[\begin{array}{c}
\mu_{1_{J}}\left[\frac{\left\{2\left(\mu_{1_{F_{1}}} \rho+a^{(1)} \delta^{(1)}\right)+\mu_{2_{G}}\right\}\left(\mu_{1_{F_{1}}} \rho+a^{(1)} \delta^{(1)}\right)}{2\left(\delta^{(1)}-\mu_{1_{G}}\right)^{3}}+\frac{\mu_{2_{F_{1}}} \rho}{2\left(\delta^{(1)}-\mu_{1_{G}}\right)^{2}}\right]\left\{t-\left(\frac{1-e^{-\left(\delta^{(1)}-\mu_{1_{G}}\right) t}}{\delta^{(1)}-\mu_{1_{G}}}\right)\right\} \\
+\mu_{1_{G}} \mu_{1_{J}}\left\{\frac{\mu_{1_{F_{1}}} \rho+a^{(1)} \delta^{(1)}}{\left(\delta^{(1)}-\mu_{1_{G}}\right)^{2}}\right\}\left\{t-\left(\frac{1-e^{-\left(\delta^{(1)}-\mu_{G}\right)} t}{\delta^{(1)}-\mu_{1_{G}}}\right)\right\} \\
-\left(a^{(1)} \delta^{(1)}+\mu_{1_{F_{1}}} \rho\right) \mu_{1_{J}}\left\{\frac{\mu_{F_{1}} \rho+a^{(1)} \delta^{(1)}}{\left(\delta^{(1)}-\mu_{1_{G}}\right)^{3}}\right\}\left\{t-\left(\frac{1-e^{-\left(\delta^{(1)}-\mu_{1_{G}}\right) t}}{\delta^{(1)}-\mu_{1_{G}}}\right)\right\}
\end{array}\right] \\
+\mu_{2_{J}}\left(\frac{\mu_{1_{F_{1}}} \rho+a^{(1)} \delta^{(1)}}{\delta^{(1)}-\mu_{1_{G}}}\right) t
\end{gathered}
$$

and the variance of the process of $L_{t}^{(2)}$ is given by

$$
\begin{gathered}
\operatorname{Var}\left(L_{t}^{(2)}\right) \\
=2 \mu_{1_{K}}\left[\begin{array}{c}
\mu_{1_{K}}\left[\frac{\left\{2\left(\mu_{1_{F}} \rho+a^{(2)} \delta^{(2)}\right)+\mu_{2_{H}}\right\}\left(\mu_{1_{F_{2}}} \rho+a^{(2)} \delta^{(2)}\right)}{2\left(\delta^{(2)}-\mu_{1_{H}}\right)^{3}}+\frac{\mu_{2_{F_{2}}} \rho}{2\left(\delta^{(2)}-\mu_{1_{H}}\right)^{2}}\right]\left\{t-\left(\frac{1-e^{-\left(\delta^{(2)}-\mu_{1_{H}}\right) t}}{\delta^{(2)}-\mu_{1_{H}}}\right)\right\} \\
+\mu_{1_{H}} \mu_{1_{K}}\left\{\frac{\mu_{F_{2}} \rho+a^{(2)} \delta^{(2)}}{\left(\delta^{(2)}-\mu_{1_{H}}\right)^{2}}\right\}\left\{t-\left(\frac{1-e^{-\left(\delta^{(2)}-\mu_{1_{H}}\right) t}}{\delta^{(2)}-\mu_{1_{H}}}\right)\right\} \\
-\left(a^{(2)} \delta^{(2)}+\mu_{1_{F_{2}}} \rho\right) \mu_{1_{K}}\left\{\frac{\mu_{1_{F_{2}}} \rho+a^{(2)} \delta^{(2)}}{\left(\delta^{(2)}-\mu_{1_{H}}\right)^{3}}\right\}\left\{t-\left(\frac{1-e^{-\left(\delta^{(2)}-\mu_{1_{H}}\right) t}}{\delta^{(2)}-\mu_{1_{H}}}\right)\right\}
\end{array}\right] \\
+\mu_{2_{K}}\left(\frac{\mu_{1_{F_{2}}} \rho+a^{(2)} \delta^{(2)}}{\delta^{(2)}-\mu_{1_{H}}}\right) t .
\end{gathered}
$$


Proof. See Appendix G.

The corresponding results for Lemma 4.1-4.2, Theorem 4.2-4.3 and Corollary 4.2 can be obtained without using the case for the stationary distribution of the process $\lambda_{t}^{(i)}(i=1,2)$. However their expressions would be very lengthy formulas with various exponential functions.

\section{Insurance application}

The proposed bivariate compound dynamic contagion process may be interpreted in the context of cyber insurance. An initial cyber attack/incident/shock (e.g. a computer virus) may be the magnitude of joint contribution to intensities for two different business risks/lines at the same time. In the bivariate compound dynamic contagion process, they are positive externally-excited joint jumps with its distribution $F\left(x^{(1)}, x^{(2)}\right), x^{(1)}>0, x^{(2)}>0$, where margins are $F_{X^{(1)}}$ and $F_{X^{(2)}}$ at the corresponding random times $\left\{T_{1, i}\right\}_{i=1,2, \ldots}$ following a Poisson process $M_{t}$ with constant rate $\rho>0$.

After-cyber attacks/incidents/shocks (e.g. infections) may be the magnitudes of contribution to intensity for each business risk/line at the different time. In the bivariate compound dynamic contagion process, they are positive self-excited jumps with distribution function $G(y), y>0$, at the corresponding random times $\left\{T_{2, j}\right\}_{j=1,2, \ldots}$ and another positive self-excited jumps with distribution function $H(z), z>0$, at the corresponding random times $\left\{T_{2, k}\right\}_{k=1,2, \ldots}$. The impact of each attack/incident/shock decays exponentially with constant rate $\delta$.

The number of losses/claims released from the first business risk/line, $N_{t}^{(1)}$ is driven by a series of after-cyber attacks/incidents/shocks $\left\{Y_{j}\right\}_{j=1,2, \ldots}$ and initial cyber attacks/incidents/shocks $\left\{X_{i}^{(1)}\right\}_{i=1,2, \cdots}$ via its intensity $\lambda_{t}^{(1)}$, and the number losses/claims released from the second business risk/line, $N_{t}^{(2)}$ is driven by a series of after-cyber attacks/incidents/shocks $\left\{Z_{k}\right\}_{k=1,2, \cdots}$ and initial cyber attacks/incidents/shocks $\left\{X_{i}^{(2)}\right\}_{i=1,2, \cdots}$ via its intensity $\lambda_{t}^{(2)}$, where initial cyber attacks/incidents/shocks $\left\{X_{i}^{(1)}, X_{i}^{(2)}\right\}_{i=1,2, \ldots}$ occur to two different business risks/lines simultaneously/collaterally with constant intensity $\rho$.

$L_{t}^{(1)}$ is the aggregate loss from the first business risk/line, where loss/claim distribution function is given by $J\left(\xi^{(1)}\right), \xi^{(1)}>0$, and $L_{t}^{(2)}$ is the aggregate loss from the second business risk/line, where loss/claim distribution function is given by $K\left(\xi^{(2)}\right), \xi^{(2)}>0$.

\subsection{Univariate case}

Set $a^{(1)}=0$ in $(2.1)$, then from (4.20) the expectation of the process $L_{t}^{(1)}$ is given by

$$
E\left(L_{t}^{(1)}\right)=\mu_{1_{J}}\left(\frac{\mu_{1_{F_{1}}} \rho}{\delta^{(1)}-\mu_{1_{G}}}\right) t, \quad \delta^{(1)}>\mu_{1_{G}}
$$

and from (4.31) its variance is given by

$$
\operatorname{Var}\left(L_{t}^{(1)}\right)
$$




$$
\begin{aligned}
& =2 \mu_{1_{J}}\left[\begin{array}{c}
\mu_{1_{J}}\left\{\frac{\left(2 \mu_{1_{F_{1}}} \rho+\mu_{2_{G}}\right) \times \mu_{1_{F_{1}}} \rho}{2\left(\delta^{(1)}-\mu_{1_{G}}\right)^{3}}+\frac{\mu_{F_{F_{1}}} \rho}{2\left(\delta^{(1)}-\mu_{\mu_{G}}\right)^{2}}\right\}\left\{t-\left(\frac{1-e^{-\left(\delta^{(1)}-\mu_{1_{G}}\right) t}}{\delta^{(1)}-\mu_{1_{G}}}\right)\right\} \\
+\mu_{1_{G}} \mu_{1_{J}}\left\{\frac{\mu_{1_{F_{1}}} \rho}{\left(\delta^{(1)}-\mu_{1_{G}}\right)^{2}}\right\}\left\{t-\left(\frac{1-e^{-\left(\delta^{(1)}-\mu_{1_{G}}\right) t}}{\delta^{(1)}-\mu_{1_{G}}}\right)\right\} \\
-\mu_{1_{J}} \mu_{1_{F_{1}}} \rho\left\{\frac{\mu_{1_{F_{1}}} \rho}{\left(\delta^{(1)}-\mu_{1_{G}}\right)^{3}}\right\}\left\{t-\left(\frac{\left.1-e^{-\left(\delta^{(1)}-\mu_{1_{G}}\right.}\right) t}{\delta^{(1)}-\mu_{1_{G}}}\right)\right\}
\end{array}\right] \\
& +\mu_{2_{J}}\left(\frac{\mu_{1_{F_{1}}} \rho}{\delta^{(1)}-\mu_{1_{G}}}\right) t
\end{aligned}
$$

If there are no self-excited jumps, from (5.1) we have

$$
\mu_{1_{J}}\left(\frac{\mu_{1_{F_{1}}} \rho}{\delta^{(1)}}\right) t,
$$

which is the expectation of compound shot-noise Cox process, and can also be found in Dassios and Jang (2003) and Jang and Fu (2012). From (5.2), the corresponding variance is given by

$$
\frac{\left(\mu_{1_{J}}\right)^{2} \mu_{2_{F_{1}}} \rho}{\left(\delta^{(1)}\right)^{2}}\left\{t-\left(\frac{1-e^{-\delta^{(1)} t}}{\delta^{(1)}}\right)\right\}+\mu_{2_{J}}\left(\frac{\mu_{1_{F_{1}}} \rho}{\delta^{(1)}}\right) t .
$$

Let us now illustrate the calculations of above expectations as cyber insurance premiums. For $F\left(x^{(1)}\right)$, we use an exponential distribution, i.e.

$$
1-e^{-\alpha x^{(1)}}, \alpha>0
$$

and for $G(y)$, we use a Loggamma distribution with probability density, i.e.

$$
\frac{\varsigma^{c}}{\psi \Gamma(c)}\left\{\ln \left(\frac{y}{\psi}+1\right)\right\}^{c-1}\left(\frac{y}{\psi}+1\right)^{-\varsigma-1}, \psi>0, \varsigma>0 \text { and } c>0
$$

to capture the effect of sudden increases of the intensity, i.e. after-cyber attacks/incidents/shocks driven by initial cyber attacks/incidents/shocks. For $J\left(\xi^{(1)}\right)$, we use a Pareto distribution with probability density, i.e.

$$
\frac{\Gamma\left(\omega^{(1)}+k^{(1)}\right)\left\{\zeta^{(1)}\right\}^{\omega^{(1)}}\left\{\xi^{(1)}\right\}^{k^{(1)}-1}}{\Gamma\left(\omega^{(1)}\right) \Gamma\left(k^{(1)}\right)\left(\zeta^{(1)}+\xi^{(1)}\right)^{\omega^{(1)}+k^{(1)}}}, \omega^{(1)}>0, \zeta^{(1)}>0 \text { and } k^{(1)}>0
$$

to accommodate catastrophic losses/claims generated from the first business risk/line due to initial and after cyber attacks/incidents/shocks. We assume interest rates to be constant.

\section{Example 5.1}

We assume that the frequency of initial cyber attack/incident/shock (e.g. a computer virus) to single business risk/line is 3 per unit time period (say, per year) with the average 
of contribution to intensity, 10. Once the virus is executed, it replicates itself by modifying other computer programs causing a series of infection to this business risk/line IT system. The mean of contribution to intensity by after-cyber attacks/incidents/shocks (e.g. infections), which are unknown at the arrival times of initial cyber attacks/incidents/shocks, is assumed to be 2.8805. We assume that the mean of catastrophic losses/claims due to initial and after cyber attacks/incidents/shocks is 12 .

Hence the parameter values to calculate the expectations are

$$
\begin{aligned}
\delta^{(1)} & =3, \quad \rho=3, \alpha=0.1, \quad \psi=1, \quad \varsigma=2.75, \quad c=3, \\
\omega^{(1)} & =3, \quad \zeta^{(1)}=4, \quad k^{(1)}=6 \text { and } t=1 .
\end{aligned}
$$

and from (5.1)-(5.4), their calculations are shown in Table 5.1.

Table 5.1

\begin{tabular}{|c|c|c|}
\hline & $\begin{array}{l}\text { Univariate compound } \\
\text { dynamic contagion process }\end{array}$ & $\begin{array}{c}\text { Univariate compound } \\
\text { shot-noise Cox process }\end{array}$ \\
\hline Mean & $3,011.71$ & 120 \\
\hline Variance & $6,713,295.5$ & 9,919 \\
\hline $\begin{array}{l}\text { Mean-standard deviation } \\
\text { principle premium }\end{array}$ & $5,602.7$ & 219.59 \\
\hline
\end{tabular}

Remark 5: Table 5.1 shows that mean-standard deviation principle premium, 5, 602.7 calculated based on (5.1)-(5.2) is extremely higher than its counterpart 219.59 calculated based on (5.3)-(5.4). It is because after-cyber attacks/incidents/shocks (e.g. infections) driven by initial cyber attacks/incidents/shocks (e.g. a computer virus). In other words, $\mu_{1_{G}}$, which is the mean of after-cyber attacks/incidents/shocks, is the main driver to raise the premium extremely higher than its counterpart. Hence the significance of after-cyber attacks/incidents/shocks driven from an initial attack/incident/shock depends on its measure $G(y)$.

Due to the digitalisation of business and economic activities, all types of risk are touched by cyber nowadays. To deal with new challenge insurers face - risks arising from cyber space, they need new tools to measure these risks. The mean-standard deviation principle premium value calculated based on (5.1)-(5.2) clearly justifies that the univariate compound dynamic contagion process can be used for modelling aggregate losses/claims from cyber attacks/incidents.

\subsection{Bivariate case}

Set $a^{(1)}=0, a^{(2)}=0$ and $\delta=\delta^{(1)}=\delta^{(2)}$, then from (4.20) and (4.21), the expectation of the process $L_{t}^{(1)}$ is given by

$$
E\left(L_{t}^{(1)}\right)=\mu_{1_{J}}\left(\frac{\mu_{1_{F_{1}}} \rho}{\delta-\mu_{1_{G}}}\right) t, \quad \delta>\mu_{1_{G}}
$$

and the expectation of the process $L_{t}^{(2)}$ is given by 


$$
E\left(L_{t}^{(2)}\right)=\mu_{1_{K}}\left(\frac{\mu_{1_{F_{2}}} \rho}{\delta-\mu_{1_{H}}}\right) t, \quad \delta>\mu_{1_{H}} .
$$

Let us assume that an insurance company charges cyber insurance premium as follows:

$$
\begin{aligned}
& E\left(L_{t}^{(1)}+L_{t}^{(2)}\right)+\phi \sqrt{\operatorname{Var}\left(L_{t}^{(1)}+L_{t}^{(2)}\right)} \\
= & E\left(L_{t}^{(1)}\right)+E\left(L_{t}^{(2)}\right)+\phi \sqrt{\operatorname{Var}\left(L_{t}^{(1)}\right)+\operatorname{Var}\left(L_{t}^{(2)}\right)+2 \operatorname{Cov}\left(L_{t}^{(1)}, L_{t}^{(2)}\right)},
\end{aligned}
$$

where $0 \leq \phi \leq 1$ and $\phi \sqrt{\operatorname{Var}\left(L_{t}^{(1)}+L_{t}^{(2)}\right)}$ can be considered as a security loading.

To calculate the covariance, we need to specify externally-excited joint jump distribution $F\left(x^{(1)}, x^{(2)}\right)$, for which we offer four choices of copulas: (1) the Farlie-Gumbel-Morgenstern (FGM) copula, (2) the Gaussian copula, (3) the $t$ copula and (4) the Gumbel copula. The Farlie-Gumbel-Morgenstern (FGM) family copula is given by

$$
C_{\theta}\left(u_{1}, u_{2}\right)=u_{1} u_{2}+\theta u_{1} u_{2}\left(1-u_{1}\right)\left(1-u_{2}\right),
$$

where $u_{1} \in[0,1], u_{2} \in[0,1]$ and $\theta \in[-1,1]$. The Gaussian family copula is given by

$$
C_{\theta}\left(u_{1}, u_{2}\right)=\Phi_{\Sigma}\left(\Phi^{-1}\left(u_{1}\right), \Phi^{-1}\left(u_{2}\right)\right),
$$

where $\Phi^{-1}$ is the inverse cumulative distribution function (c.d.f.) of a standard univariate normal, $\Phi_{\Sigma}$ denotes the c.d.f. for a bivariate normal distribution with mean vector zero and covariance matrix $\Sigma$, where $\Sigma$ the $2 \times 2$ matrix with 1 on the diagonal and correlation coefficient $\theta$ otherwise, $u_{1} \in[0,1], u_{2} \in[0,1]$ and $\theta \in[-1,1]$. The $t$ copula is given by

$$
C_{\theta}\left(u_{1}, u_{2}\right)=t_{\varepsilon, \Sigma}\left(t_{\nu}^{-1}\left(u_{1}\right), t_{\nu}^{-1}\left(u_{2}\right)\right),
$$

where $t_{\nu}^{-1}$ is the inverse cumulative distribution function (c.d.f.) of a standard univariate $t$, $t_{\varepsilon, \Sigma}$ denotes the c.d.f. for a bivariate $t$ distribution with mean vector zero and covariance matrix $\Sigma$, where $\Sigma$ the $2 \times 2$ matrix with 1 on the diagonal and correlation coefficient $\theta$ otherwise, $\varepsilon$ is the degrees of freedom, $u_{1} \in[0,1], u_{2} \in[0,1]$ and $\theta \in[-1,1]$. The Gumbel copulas are given by

$$
C_{\theta}\left(u_{1}, u_{2}\right)=\exp \left[\left\{\left(-\ln \left(u_{1}\right)\right)^{-\theta}+\left(-\ln \left(u_{2}\right)\right)^{-\theta}-1\right\}^{-\frac{1}{\theta}}\right],
$$

where $u_{1} \in[0,1], u_{2} \in[0,1]$ and $\theta \in[1, \infty)$.

For $F\left(x^{(2)}\right)$, we also use an exponential distribution, i.e.

$$
F\left(x^{(2)}\right)=1-e^{-\beta x^{(2)}}(\beta>0)
$$

and for $H(z)$, we use a Fréchet distribution with probability density, i.e.

$$
\frac{\epsilon}{\varphi}\left(\frac{z}{\varphi}\right)^{-\epsilon-1} e^{-\left(\frac{z}{\varphi}\right)^{-\epsilon}}, \varphi>0 \text { and } \epsilon>0
$$


to capture the effect of sudden increases of the intensity, i.e. after-cyber attacks/incidents/shocks driven by initial cyber attacks/incidents/shocks. For $K\left(\xi^{(2)}\right)$, we use another Pareto distribution with probability density, i.e.

$$
\frac{\Gamma\left(\omega^{(2)}+k^{(2)}\right)\left\{\zeta^{(2)}\right\}^{\omega^{(2)}}\left\{\xi^{(2)}\right\}^{k^{(2)}-1}}{\Gamma\left(\omega^{(2)}\right) \Gamma\left(k^{(2)}\right)\left(\zeta^{(2)}+\xi^{(2)}\right)^{\omega^{(2)}+k^{(2)}}}, \omega^{(2)}>0, \zeta^{(2)}>0 \text { and } k^{(2)}>0
$$

to accommodate catastrophic losses/claims generated from the second business risk/line due to initial and after cyber attacks/incidents/shocks.

For the next four examples, we assume that the frequency of initial joint cyber attack/incident/shock (e.g. a computer virus) to two business risks/lines is 3 per unit time period (say, per year) with the same average of contributions to both intensities, 10 . Once the virus is executed, it replicates itself by modifying other computer programs causing a series of infection to two business risks/lines IT systems, separately. The mean of contribution to the first \& second business risk/line intensity by after-cyber attacks/incidents/shocks (e.g. infections), which are unknown at the arrival times of initial cyber attacks/incidents/shocks, is assumed to be 2.8805 and 2.7082, respectively. We assume that the mean of catastrophic losses/claims from two business risks/lines due to initial and after cyber attacks/incidents/shocks is 12 and 8, respectively. As the security loading factor, this insurance company uses 1.

Hence the parameter values used to calculate cyber loss insurance premiums are

$$
\begin{aligned}
\beta & =0.1, \quad \varphi=2, \quad \epsilon=3, \phi=1, \\
\omega^{(2)} & =4, \quad \zeta^{(2)}=4 \text { and } k^{(2)}=6
\end{aligned}
$$

and using the parameter values in Example 5.1, let us now illustrate the calculations of cyber loss insurance premiums at different value of $\theta$, comparing their counterparts when there are no after cyber attacks/incidents/shocks.

Example 5.2 (FGM copula)

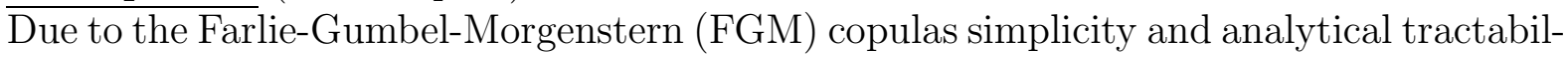
ity, we have

$$
\mu_{1_{F_{1,2}}}=\int_{0}^{\infty} \int_{0}^{\infty} x^{(1)} x^{(2)} d F\left(x^{(1)}, x^{(2)}\right)=\frac{1}{\alpha \beta}\left(1+\frac{\theta}{4}\right)
$$

\begin{tabular}{|c|c|c|}
\hline \multicolumn{3}{|c|}{ Table 5.2} \\
\hline$\theta$ & $\begin{array}{l}\text { Bivariate compound } \\
\text { dynamic contagion process }\end{array}$ & $\begin{array}{l}\text { Bivariate compound } \\
\text { shot-noise Cox process }\end{array}$ \\
\hline-1 & 6481.74 & 331.28 \\
\hline-0.5 & 6484.83 & 333.34 \\
\hline 0 & 6487.92 & 335.38 \\
\hline 0.5 & 6491.01 & 337.38 \\
\hline 1 & 6494.09 & 339.36 \\
\hline
\end{tabular}

to calculate $E\left(L_{t}^{(1)} L_{t}^{(2)}\right)$ in $\operatorname{Cov}\left(L_{t}^{(1)}, L_{t}^{(2)}\right)$. Cyber loss insurance premium calculations are shown in Table 5.2, 
where for bivariate compound dynamic contagion case, we have

$$
\begin{aligned}
& E\left(L_{t}^{(1)}\right)=3011.71 \text { and } \operatorname{Var}\left(L_{t}^{(1)}\right)=6,713,296 \\
& E\left(L_{t}^{(2)}\right)=822.582 \text { and } \operatorname{Var}\left(L_{t}^{(2)}\right)=197,473
\end{aligned}
$$

and for bivariate compound shot-noise case, we have

$$
\begin{aligned}
& E\left(L_{t}^{(1)}\right)=120 \text { and } \operatorname{Var}\left(L_{t}^{(1)}\right)=9,919.32 \\
& E\left(L_{t}^{(2)}\right)=80 \text { and } \operatorname{Var}\left(L_{t}^{(2)}\right)=4,035.25
\end{aligned}
$$

The covariances between $L_{t}^{(1)}$ and $L_{t}^{(2)}$ and their corresponding linear correlation coefficients at different value of $\theta$, compared to their counterparts when there are no self-excited jumps are shown in Table 5.3 and Table 5.4, respectively.

\begin{tabular}{|l|c|c|}
\hline \multicolumn{2}{|c|}{ Table $\mathbf{5 . 3} \operatorname{Cov}\left(L_{t}^{(1)}, L_{t}^{(2)}\right)$} \\
\hline \multicolumn{3}{|c|}{} \\
\hline$\theta$ & $\begin{array}{l}\text { Bivariate compound } \\
\text { dynamic contagion process }\end{array}$ & $\begin{array}{l}\text { Bivariate compound } \\
\text { shot-noise Cox process }\end{array}$ \\
\hline-1 & 49123.16 & 1639.83 \\
\hline-0.5 & 57310.35 & 1913.13 \\
\hline 0 & 65497.54 & 2186.44 \\
\hline 0.5 & 73684.73 & 2459.74 \\
\hline 1 & 81871.93 & 2733.05 \\
\hline
\end{tabular}

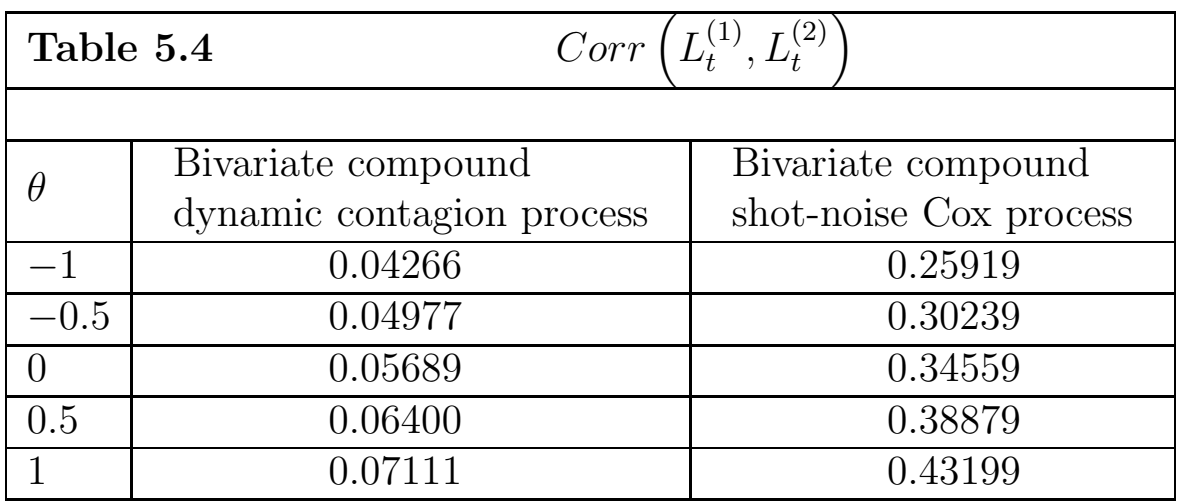

Example 5.2 (Gaussian copula)

For the Gaussian copulas, using the programming language $\mathrm{R}$ cyber loss insurance premium calculations are shown in Table 5.5, 


\begin{tabular}{|l|c|c|}
\hline \multicolumn{3}{|c|}{ Table 5.5 Cyber loss insurance premium } \\
\hline$\theta$ & $\begin{array}{l}\text { Bivariate compound } \\
\text { dynamic contagion process }\end{array}$ & $\begin{array}{l}\text { Bivariate compound } \\
\text { shot-noise Cox process }\end{array}$ \\
\hline-0.99 & $6,472.08$ & 324.61 \\
\hline-0.5 & $6,478.91$ & 329.36 \\
\hline 0 & $6,487.92$ & 335.38 \\
\hline 0.5 & $6,499.08$ & 342.51 \\
\hline 0.99 & $6,512.20$ & 350.49 \\
\hline
\end{tabular}

The covariances between $L_{t}^{(1)}$ and $L_{t}^{(2)}$ and their corresponding linear correlation coefficients at different value of $\theta$, compared to their counterparts when there are no self-excited jumps are shown in Table 5.6 and Table 5.7, respectively.

\begin{tabular}{|l|c|c|}
\hline \multicolumn{2}{|c|}{ Table 5.6 $\operatorname{Cov}\left(L_{t}^{(1)}, L_{t}^{(2)}\right)$} \\
\hline \multicolumn{3}{|c|}{} \\
\hline$\theta$ & $\begin{array}{l}\text { Bivariate compound } \\
\text { dynamic contagion process }\end{array}$ & $\begin{array}{l}\text { Bivariate compound } \\
\text { shot-noise Cox process }\end{array}$ \\
\hline-0.99 & $23,571.72$ & 786.87 \\
\hline-0.5 & $41,632.69$ & $1,389.78$ \\
\hline 0 & $65,497.54$ & $2,186.44$ \\
\hline 0.5 & $95,172.84$ & $3,177.06$ \\
\hline 0.99 & $130,216.13$ & $4,346.88$ \\
\hline
\end{tabular}

\begin{tabular}{|l|c|c|}
\hline \multicolumn{2}{|c|}{ Table $\mathbf{5 . 7} \operatorname{Corr}\left(L_{t}^{(1)}, L_{t}^{(2)}\right)$} \\
\hline \multicolumn{3}{|c|}{} \\
\hline$\theta$ & $\begin{array}{l}\text { Bivariate compound } \\
\text { dynamic contagion process }\end{array}$ & $\begin{array}{l}\text { Bivariate compound } \\
\text { shot-noise Cox process }\end{array}$ \\
\hline-0.99 & 0.02047 & 0.12437 \\
\hline-0.5 & 0.03616 & 0.21967 \\
\hline 0 & 0.05689 & 0.34559 \\
\hline 0.5 & 0.08266 & 0.50217 \\
\hline 0.99 & 0.11309 & 0.68707 \\
\hline
\end{tabular}

Example 5.3 ( $t$ copula with $\varepsilon=5)$

For the $t$ copulas, using the programming language $\mathrm{R}$ cyber loss insurance premium calculations are shown in Table 5.8

\begin{tabular}{|l|c|c|}
\hline \multicolumn{3}{|c|}{ Table 5.8 Cyber loss insurance premium } \\
\hline$\theta$ & $\begin{array}{l}\text { Bivariate compound } \\
\text { dynamic contagion process }\end{array}$ & $\begin{array}{l}\text { Bivariate compound } \\
\text { shot-noise Cox process }\end{array}$ \\
\hline-0.99 & $6,472.08$ & 324.62 \\
\hline-0.5 & $6,479.53$ & 329.78 \\
\hline 0 & $6,488.87$ & 336.00 \\
\hline 0.5 & $6,499.76$ & 342.93 \\
\hline 0.99 & $6,512.21$ & 350.50 \\
\hline
\end{tabular}


The covariances between $L_{t}^{(1)}$ and $L_{t}^{(2)}$ and their corresponding linear correlation coefficients at different value of $\theta$, comparing their counterparts when there are no self-excited jumps are shown in Table 5.9 and Table 5.10, respectively.

\begin{tabular}{|c|c|c|}
\hline \multicolumn{3}{|c|}{ Table 5.9} \\
\hline$\theta$ & $\begin{array}{l}\text { Bivariate compound } \\
\text { dynamic contagion process }\end{array}$ & $\begin{array}{l}\text { Bivariate compound } \\
\text { shot-noise Cox process }\end{array}$ \\
\hline-0.99 & $23,595.21$ & 787.66 \\
\hline-0.5 & $43,268.73$ & $1,444.40$ \\
\hline 0 & $68,008.96$ & $2,270.28$ \\
\hline 0.5 & $96,986.52$ & $3,237.61$ \\
\hline 0.99 & $130,248.18$ & $4,347.95$ \\
\hline
\end{tabular}

\begin{tabular}{|l|c|c|}
\hline \multicolumn{2}{|c|}{ Table $\mathbf{5 . 1 0} \operatorname{Corr}\left(L_{t}^{(1)}, L_{t}^{(2)}\right)$} \\
\hline \multicolumn{3}{|c|}{} \\
\hline$\theta$ & $\begin{array}{l}\text { Bivariate compound } \\
\text { dynamic contagion process }\end{array}$ & $\begin{array}{l}\text { Bivariate compound } \\
\text { shot-noise Cox process }\end{array}$ \\
\hline-0.99 & 0.02049 & 0.12450 \\
\hline-0.5 & 0.03758 & 0.22830 \\
\hline 0 & 0.05907 & 0.35884 \\
\hline 0.5 & 0.08423 & 0.51174 \\
\hline 0.99 & 0.11312 & 0.68724 \\
\hline
\end{tabular}

Example 5.4 (Gumbel copula)

For the Gaussian copulas, using the programming language $\mathrm{R}$ cyber loss insurance premium calculations are shown in Table 5.11.

\begin{tabular}{|l|c|c|}
\hline \multicolumn{2}{|c|}{ Table 5.11 Cyber loss insurance premium } \\
\hline \multicolumn{3}{|c|}{} \\
\hline$\theta$ & $\begin{array}{l}\text { Bivariate compound } \\
\text { dynamic contagion process }\end{array}$ & $\begin{array}{c}\text { Bivariate compound } \\
\text { shot-noise Cox process }\end{array}$ \\
\hline 1.001 & $6,487.97$ & 335.41 \\
\hline 2 & $6,506.88$ & 347.30 \\
\hline 5 & $6,511.66$ & 350.17 \\
\hline 10 & $6,512.29$ & 350.55 \\
\hline 100 & $6,512.49$ & 350.67 \\
\hline
\end{tabular}

The covariances between $L_{t}^{(1)}$ and $L_{t}^{(2)}$ and their corresponding linear correlation coefficients at different value of $\theta$, compared to their counterparts when there are no self-excited jumps are shown in Table 5.12 and Table 5.13, respectively. 


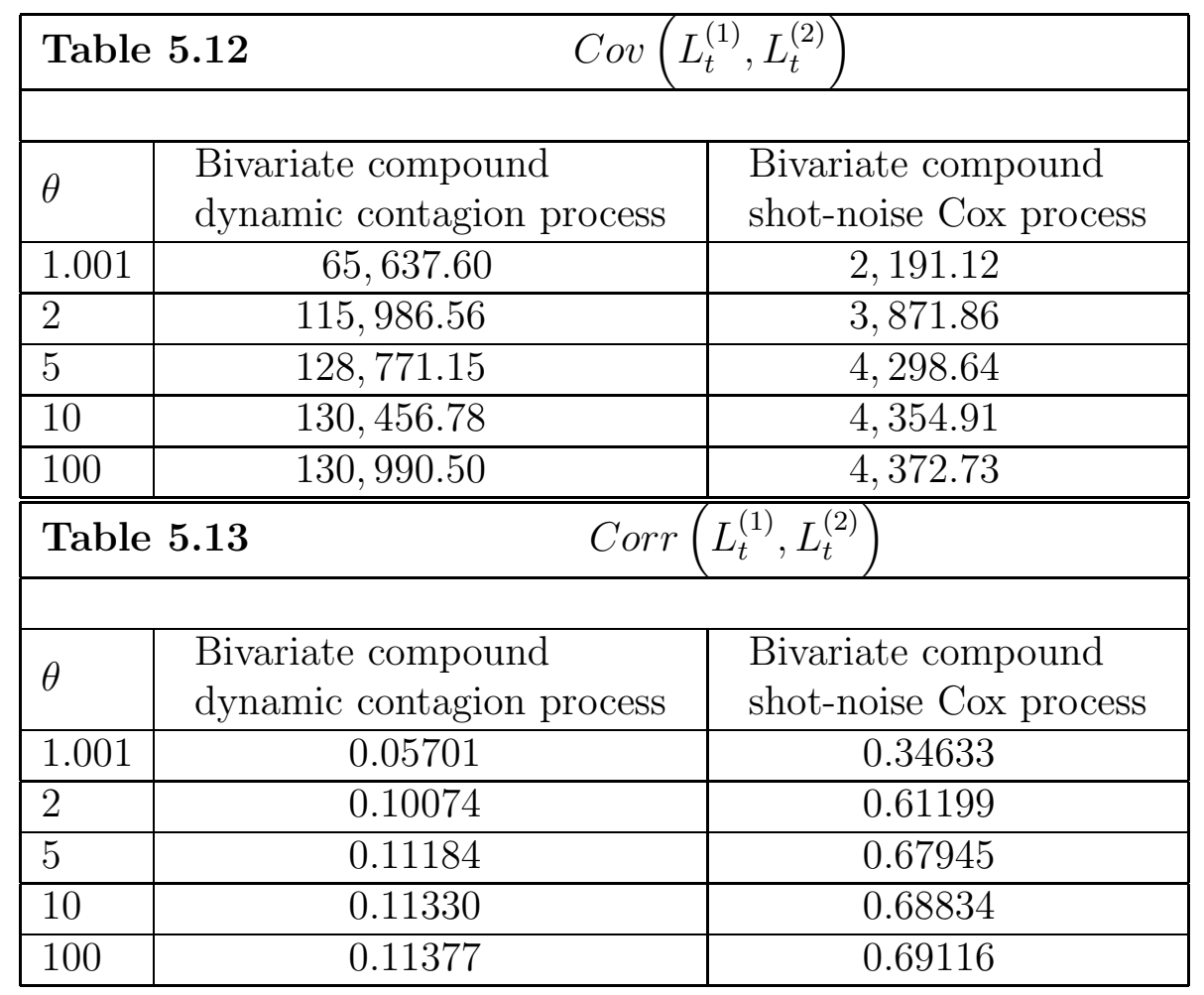

Remark 6: Table 5.2, 5.5, 5.8 and 5.11 show that cyber loss insurance premium values calculated using the bivariate compound dynamic contagion process are significantly higher than their counterparts calculated using the bivariate compound shot-noise Cox process at a different value of $\theta$. The covariances in Table 5.3, 5.6, 5.9 and 5,12 also support this. It is because two means for after-cyber attacks/incidents/shocks, i.e. $\mu_{1_{G}}$ and $\mu_{1_{H}}$, and $\mu_{1_{F_{1,2}}}$ are involved in calculating cyber loss insurance premium values using (5.7). Hence the significance of two separate after-cyber attacks/incidents/shocks impacts driven from initial joint cyber attack/incident/shock depends on two measures $G(y)$ and $H(z)$. It will be of interest to examine cyber loss insurance premium values using other joint measures for initial cyber attack/incident/shock as well as other measures for after-cyber attacks/incidents/shocks.

Remark 7: Table 5.4, 5.7, 5.10 and 5.13 show that the linearities between $L_{t}^{(1)}$ and $L_{t}^{(2)}$ calculated using the bivariate compound dynamic contagion process and the bivariate compound shot-noise Cox process at a different value of $\theta$. They show the former linearities between $L_{t}^{(1)}$ and $L_{t}^{(2)}$ significantly lower than the latter linearities between $L_{t}^{(1)}$ and $L_{t}^{(2)}$. It is because two separate after-cyber attacks/incidents/shocks weaken the linearity between $L_{t}^{(1)}$ and $L_{t}^{(2)}$. Therefore it will be also of interest to compare bivariate distribution for compound dynamic contagion case with its counterpart, in particular seeing their two tail corners inverting bivariate Fast Fourier transform using bivariate Laplace transform of the process $\left(L_{t}^{(1)}, L_{t}^{(2)}\right)$ shown in Section 3.

To make easier for statistical analysis, further business applications and research, we close this section providing the simulation algorithm for one sample path of the bivariate compound dynamic contagion process $\left(\left(\begin{array}{c}L_{t}^{(1)} \\ L_{t}^{(2)}\end{array}\right)\left(\begin{array}{c}N_{t}^{(1)} \\ N_{t}^{(2)}\end{array}\right),\left(\begin{array}{c}\lambda_{t}^{(1)} \\ \lambda_{t}^{(2)}\end{array}\right)\right)$, with $m$ jump times 
$\left[\begin{array}{c}\left\{T_{1}^{*(1)}, T_{2}^{*(1)}, \cdots, T_{m}^{*(1)}\right\} \\ \left\{T_{1}^{*(2)}, T_{2}^{*(2)}, \cdots, T_{m}^{*(2)}\right\}\end{array}\right]$ in the process $\left(\begin{array}{c}\lambda_{t}^{(1)} \\ \lambda_{t}^{(2)}\end{array}\right)$ (see Figure 1). This algorithm has been extended from Dassios and Zhao (2011) Section 5 algorithm, where they have shown how to simulate the univariate dynamic contagion process.

Algorithm 5.1. (The bivariate compound dynamic contagion process simulation algorithm)

1. Set the initial conditions $T_{0}^{*(1)}=T_{0}^{*(2)}=0, \lambda_{T_{0}^{*(1)+}}^{(1)}=\lambda_{0}^{(1)}>a^{(1)}, \lambda_{T_{0}^{*(2)+}}^{(2)}=$ $\lambda_{0}^{(2)}>a^{(2)}$ and $i \in\{0,1,2, \ldots, m-1\}$.

2. Simulate the $(i+1)^{\text {th }}$ externally excited joint jump waiting time $E_{i+1}^{*}$ by

$$
E_{i+1}^{*}=-\frac{1}{\rho} \ln U, \quad U \sim \mathrm{U}[0,1]
$$

3. (i) Simulate the $(i+1)^{\text {th }}$ self-excited jump waiting time $S_{i+1}^{*(1)}$ by

$$
S_{i+1}^{*(1)}=\left\{\begin{array}{ll}
S_{1, i+1}^{*(1)} \wedge S_{2, i+1}^{*(1)} & \left(d_{i+1}^{(1)}>0\right. \\
S_{2, i+1}^{*(1)} & \left(d_{i+1}^{(1)}<0\right.
\end{array}\right)
$$

where

$$
d_{i+1}^{(1)}=1+\frac{\delta^{(1)} \ln U_{11}}{\lambda_{T_{i}^{*(1)+}}^{(1)}-a^{(1)}}, \quad U_{11} \sim \mathrm{U}[0,1]
$$

and

$$
S_{1, i+1}^{*(1)}=-\frac{1}{\delta^{(1)}} \ln d_{i+1}^{(1)} ; \quad S_{2, i+1}^{*(1)}=-\frac{1}{a^{(1)}} \ln U_{12}, \quad U_{12} \sim \mathrm{U}[0,1] .
$$

(ii) Similarly, simulate the $(i+1)^{\text {th }}$ self-excited jump waiting time $S_{i+1}^{*(2)}$ by

$$
S_{i+1}^{*(2)}=\left\{\begin{array}{ll}
S_{1, i+1}^{*(2)} \wedge S_{2, i+1}^{*(2)} & \left(d_{i+1}^{(2)}>0\right. \\
S_{2, i+1}^{*(2)} & \left(d_{i+1}^{(2)}<0\right.
\end{array}\right)
$$

where

$$
d_{i+1}^{(2)}=1+\frac{\delta^{(2)} \ln U_{21}}{\lambda_{T_{i}^{(2)+}}^{(2)}-a^{(2)}}, \quad U_{21} \sim \mathrm{U}[0,1]
$$

and 


$$
S_{1, i+1}^{*(2)}=-\frac{1}{\delta^{(2)}} \ln d_{i+1}^{(2)} ; \quad S_{2, i+1}^{*(2)}=-\frac{1}{a^{(2)}} \ln U_{22}, \quad U_{22} \sim \mathrm{U}[0,1] .
$$

4. Simulate the $(i+1)^{\text {th }}$ overall jump time $T_{i+1}^{*}$ by

$$
T_{i+1}^{*}=T_{i}^{*}+S_{i+1}^{*(1)} \wedge S_{i+1}^{*(2)} \wedge E_{i+1}^{*}, \text { where } T_{0}^{*}=T_{0}^{*(1)}=T_{0}^{*(2)}=0
$$

5. (i) The $(i+1)^{\text {th }}$ jump time for the process $\lambda_{t}^{(1)}$ is given by the overall jump time $T_{i+1}^{*}$ in Step 4, i.e.

$$
T_{i+1}^{*(1)}=T_{i+1}^{*}=\left\{\begin{array}{ll}
T_{i}^{*}+S_{i+1}^{*(1)} & \left(S_{i+1}^{*(1)} \wedge S_{i+1}^{*(2)} \wedge E_{i+1}^{*}=S_{i+1}^{*(1)}\right) \\
T_{i}^{*}+E_{i+1}^{*} & \left(S_{i+1}^{*(1)} \wedge S_{i+1}^{*(2)} \wedge E_{i+1}^{*}=E_{i+1}^{*}\right)
\end{array},\right.
$$

where $S_{i+1}^{*(1)} \wedge S_{i+1}^{*(2)} \wedge E_{i+1}^{*}=S_{i+1}^{*(2)}$ is irrelevant to the $(i+1)^{\text {th }}$ jump time for the process $\lambda_{t}^{(1)}$.

(ii) Similarly, the $(i+1)^{\text {th }}$ jump time for the process $\lambda_{t}^{(2)}$ is given by the overall jump time $T_{i+1}^{*}$ in Step 4, i.e.

$$
T_{i+1}^{*(2)}=T_{i+1}^{*}= \begin{cases}T_{i}^{*}+S_{i+1}^{*(2)} & \left(S_{i+1}^{*(1)} \wedge S_{i+1}^{*(2)} \wedge E_{i+1}^{*}=S_{i+1}^{*(2)}\right) \\ T_{i}^{*}+E_{i+1}^{*} & \left(S_{i+1}^{*(1)} \wedge S_{i+1}^{*(2)} \wedge E_{i+1}^{*}=E_{i+1}^{*}\right)\end{cases}
$$

where $S_{i+1}^{*(1)} \wedge S_{i+1}^{*(2)} \wedge E_{i+1}^{*}=S_{i+1}^{*(1)}$ is irrelevant to the $(i+1)^{\text {th }}$ jump time for the process $\lambda_{t}^{(2)}$.

6. The changes at jump time $T_{i+1}^{*(1)}$ in the intensity process $\lambda_{t}^{(1)}$ is given by

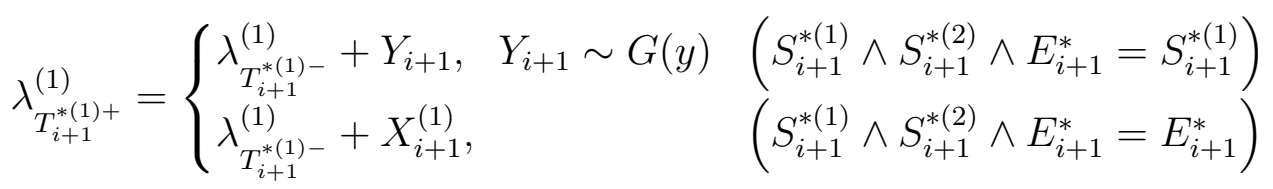

and the changes at jump time $T_{i+1}^{*(2)}$ in the intensity process $\lambda_{t}^{(2)}$ is given by

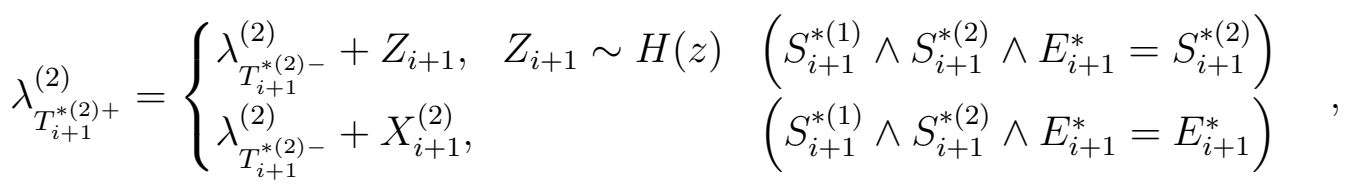

where

$$
\lambda_{T_{i+1}^{*(1)-}}^{(1)}=\left(\lambda_{T_{i}^{*(1)+}}^{(1)}-a^{(1)}\right) e^{-\delta^{(1)}\left(T_{i+1}^{*(1)}-T_{i}^{*(1)}\right)}+a^{(1)},
$$




$$
\begin{gathered}
\lambda_{T_{i+1}^{*(2)-}}^{(2)}=\left(\lambda_{T_{i}^{*(2)+}}^{(2)}-a^{(2)}\right) e^{-\delta^{(2)}\left(T_{i+1}^{*(2)}-T_{i}^{*(2)}\right)}+a^{(2)}, \\
\left(X_{i+1}^{(1)}, X_{i+1}^{(2)}\right) \sim F\left(x^{(1)}, x^{(2)}\right),
\end{gathered}
$$

where the joint distribution of the vector $\left(X_{i+1}^{(1)}, X_{i+1}^{(2)}\right)$ is assumed to be of the form $C_{\theta}\left(F\left(x^{(1)}\right), F\left(x^{(2)}\right)\right)$ with $C_{\theta}$ being a given copula.

7. The change at jump time $T_{i+1}^{*(1)}$ in the point process $N_{t}^{(1)}$ is given by

$$
N_{T_{i+1}^{*(1)+}}^{(1)}= \begin{cases}N_{T_{i+1}^{*(1)-}+1}^{(1)} & \left(S_{i+1}^{*(1)} \wedge S_{i+1}^{*(2)} \wedge E_{i+1}^{*}=S_{i+1}^{*(1)}\right) \\ N_{T_{i+1}^{*(1)}}^{(1)} & \left(S_{i+1}^{*(1)} \wedge S_{i+1}^{*(2)} \wedge E_{i+1}^{*}=E_{i+1}^{*}\right)\end{cases}
$$

and the change at jump time $T_{i+1}^{*(2)}$ in the point process $N_{t}^{(2)}$ is given by

$$
N_{T_{i+1}^{*(2)+}}^{(2)}= \begin{cases}N_{T_{i+1}^{*(2)-}}^{(2)}+1 & \left(S_{i+1}^{*(1)} \wedge S_{i+1}^{*(2)} \wedge E_{i+1}^{*}=S_{i+1}^{*(2)}\right) \\ N_{T_{i+1}^{(2)}}^{*(2)-} & \left(S_{i+1}^{*(1)} \wedge S_{i+1}^{*(2)} \wedge E_{i+1}^{*}=E_{i+1}^{*}\right)\end{cases}
$$

8. The change at jump time $T_{i+1}^{*(1)}$ in the compound point process $L_{t}^{(1)}$ is given by

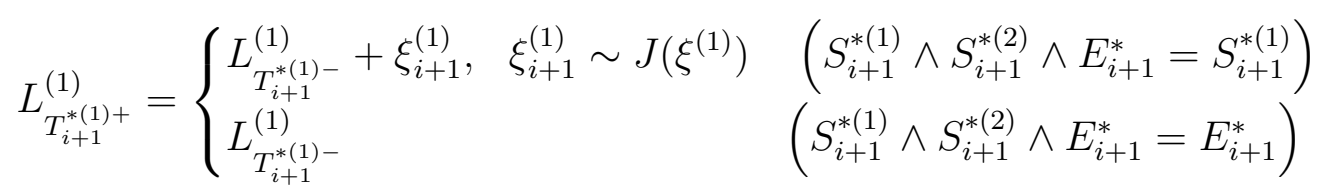

and the change at jump time $T_{i+1}^{*(2)}$ in the compound point process $L_{t}^{(2)}$ is given by

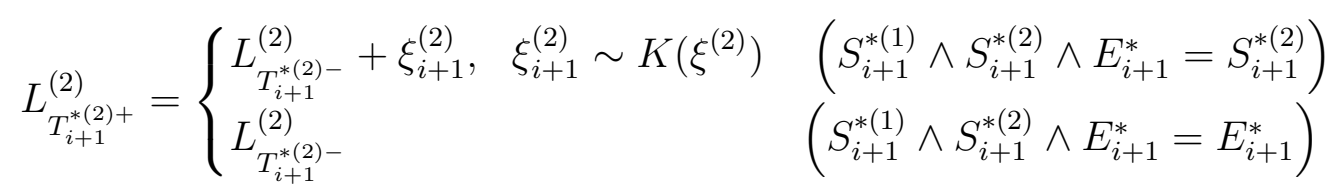

\section{Conclusion}

Digitalisation of business and economic activities have changed the risk landscape to cyber space. A cyber attack can trigger multiple, catastrophic and contagious losses to corporates and governments due to IT system interdependence. It is a real threat to all organisations as the number of cyber attacks and its complex way of doing so are rising.

Cyber insurance can be purchased to cover economic and financial losses occurring from cyber incidents. However, due to the complexity of cyber risks, i.e. multiple, catastrophic 

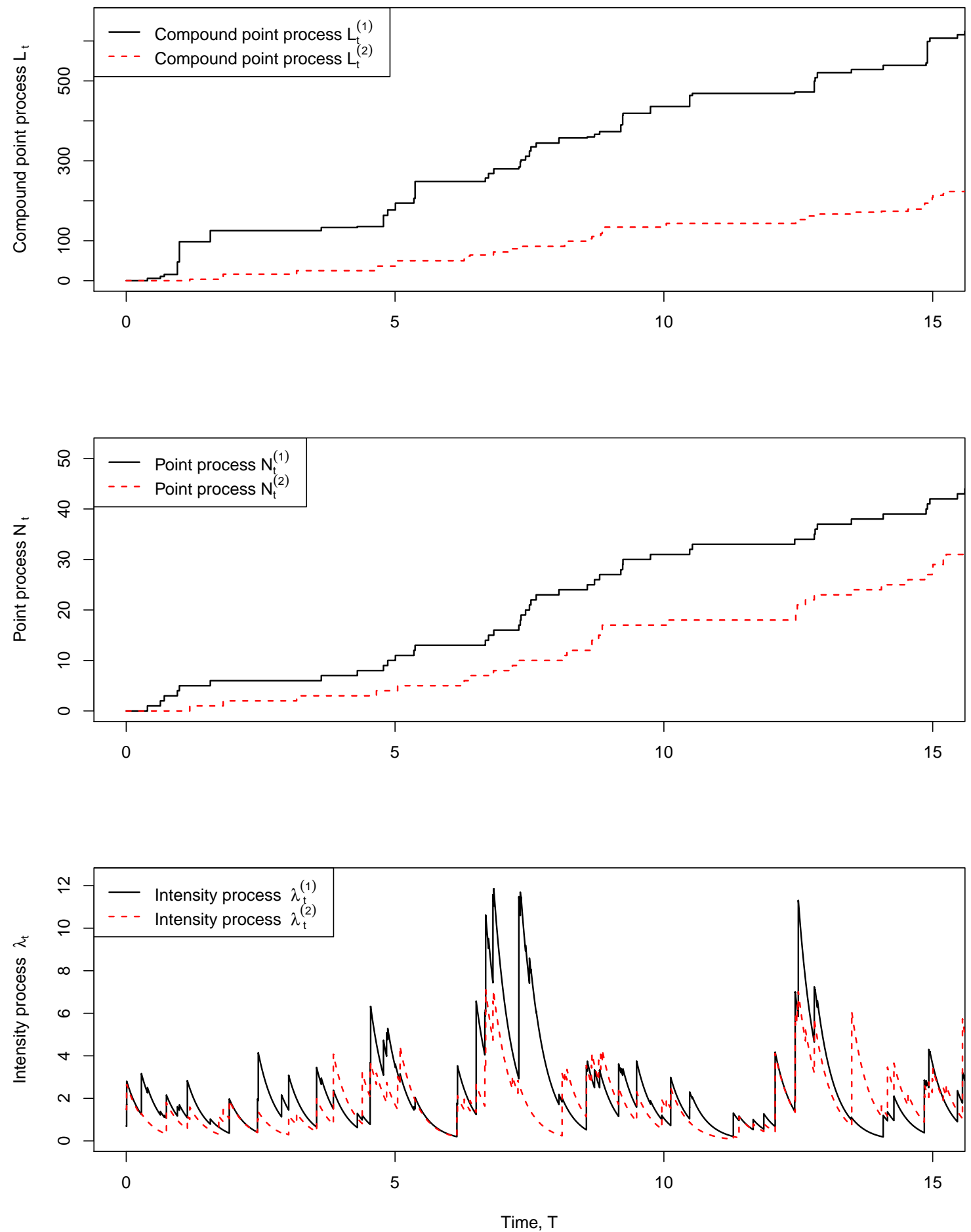

Figure 1: Simulated sample path of the bivariate compound dynamic contagion process: Intensity process $\left(\lambda_{t}^{(1)}, \lambda_{t}^{(2)}\right)$, point processes $\left(N_{t}^{(1)}, N_{t}^{(2)}\right)$, and compound point processes $\left(L_{t}^{(1)}, L_{t}^{(2)}\right)$. FGM copula is considered with parameter $\theta$. The parameters for the process 1 and process 2 are $\left(a^{(1)}, a^{(2)}, \rho, \delta^{(1)}, \delta^{(2)} ; \alpha, \psi, \varsigma, c ; \beta, \varphi, \epsilon ; \theta ; \omega^{(1)}, \omega^{(2)}\right.$, $\left.\zeta^{(1)}, \zeta^{(2)}, k^{(1)}, k^{(2)} ; \lambda_{0}^{(1)}, \lambda_{0}^{(2)}\right)=(0,0,3,3,3 ; 0.5,1,11,3 ; 1,0.5,15 ; 0.5 ; 3,4,4,4,6,6 ; 0.7,1.5)$. 
and contagious losses, it is difficult for insurers to price cyber insurance products. To provide insurers with a tool to deal with the ongoing challenge of new risks, we introduce a bivariate compound dynamic contagion process, which accommodate the interdependence of IT system and the frequency and impact of cyber events.

Our numerical results confirm that cyber loss insurance premiums calculated using the bivariate compound dynamic contagion process are significantly higher than their counterparts calculated using a bivariate compound shot-noise Cox process. For that purpose, we provided moment-based insurance premium calculations using a log gamma distribution and a Fréchet distribution for two separate self-excited jumps (i.e. after-cyber attacks/incidents/shocks), two different exponential distributions and four different copulas (i.e. the Farlie-Gumbel-Morgenstern (FGM) family copula, the Gaussian family copula, the $t$ copula and the Gumbel copula) for externally excited joint jumps (i.e. initial cyber attacks/incidents/shocks). Two Pareto distributions were used to represent catastrophic cyber losses from contagious cyber attacks. This suggests that the bivariate compound dynamic contagion process can be considered for modelling two aggregate cyber losses to calculate cyber loss insurance premiums accommodating waves of events and with the critical aspects of the interdependence of IT system and the impact of cyber events taken into account. For further research, we may consider the extension of dimension, other copulas, other measures for initial and after cyber attacks/incidents/shocks, and other measures for cyber losses. As loss/claim size and after-cyber attacks/incidents/shocks (self-exciting jumps) could be correlated, considering the dependency $\left\{Y_{j}\right\}_{j=1,2, \cdots}$ and $\left\{\Xi_{j}^{(1)}\right\}_{j=1,2, \cdots}$ and $\left\{Z_{k}\right\}_{k=1,2, \cdots}$ and $\left\{\Xi_{k}^{(1)}\right\}_{k=1,2, \cdots}$, respectively could be another object of further research.

Cyber attacks would occur more often as all types of risk are touched by cyber space due to digitalisation of business and economic activities, so the proposed bivariate compound dynamic contagion process can be an improved model for insurance companies to quantify cyber losses. The bivariate compound dynamic contagion process is also very much applicable to credit, insurance, market and other operational risks. We hope that what we presented in this paper provides practitioners with feasible models to quantify cyber losses, and to deal with a variety of problems in economics, finance and insurance.

\section{Acknowledgements}

Rosy Oh's research was supported by Basic Science Research Program through the National Research Foundation of Korea(NRF) funded by the Ministry of Education (Grant No. 2019R1A6A1A11051177 and 2020R1I1A1A01067376).

\section{References}

Allianz (2016), Allianz Risk Barometer Top Business Risks.

Aït-Sahalia, Y., Cacho-Diaz, J. A. and Laeven, R. J. (2015) : Modeling financial contagion using mutually exciting jump processes, Journal of FinancialEconomics $117(3), 585-606$.

Ait-Sahalia, Y., Laeven, R. J. and Pelizzon, L. (2014) : Mutual excitation in Eurozone sovereign CDS, Journal of Econometrics, Journal of FinancialEconomics 183(2), 151-167. 
Bauwens, L. and Hautsch, N. (2009). Modelling Financial High Frequency Data Using Point Processes, In: Handbook of Financial Time Series, T.G. Andersen, R.A. Davis, J.-P. Kreiss and T. Mikosch (eds), Springer.

Biener, C., Eling, M. and Wirfs, J. H. (2015): Insurability of Cyber Risk: An Empirical Analysis, The Geneva Papers on Risk and Insurance - Issues and Practice, 40(1), 131-158.

Bowsher, C. G. (2007) : Modelling security market events in continuous time: Intensity based, multivariate point process models, Journal of Econometrics, 141(2), 876-912.

Chavez-Demoulin, V., Davison, A. C. and McNeil, A. J. (2005): Estimating Value-at-Risk: A point process approach, Quantitative Finance, 5(2), 227-234.

Dassios, A. and Jang, J. (2003) : Pricing of catastrophe reinsurance \& derivatives using the Cox process with shot noise intensity, Finance \& Stochastics, 7/1, 7395.

Dassios, A. and Zhao, H. (2011) : A dynamic contagion process, Advances in Applied Probability, 43, 814-846.

Dassios, A. and Zhao, H. (2012) : Ruin by Dynamic Contagion Claims, Insurance: Mathematics \& Economics, 51/1, 93-106.

Dassios, A. and Zhao, H. (2017a) : A generalized contagions process with an application to credit risk, International Journal of Theoretical and Applied Finance, 20(1), 1750003 (33 pages).

Dassios, A. and Zhao, H. (2017b) : Efficient simulation of clustering jumps with CIR intensity, Operations Research, 65(6), 1494-1515.

Davis, M. H. A.(1984) : Piecewise deterministic Markov processes: A general class of non diffusion stochastic models. J. R. Stat. Soc. B 46, 353-388.

Dong, X. (2014): Compensators and diffusion approximation of point processes and applications, Ph.D Thesis. Imperial College London.

Embrechts, P., Liniger, T. and Lin, L. (2011) : Multivariate Hawkes Processes: an Application to Financial Data, Journal of Applied Probability, pecial Volume 48(A), 367-378

Errais, E., Giesecke, K. and Goldberg, L. R. (2010) : Affine Point Processes and Portfolio Credit Risk, SIAM Journal on Financial Mathematics, 1, 642-665.

Gao, X, Zhou, X and Zhu, L. (2018) : Transform analysis for Hawkes processes with applications in dark pool trading, Quantitative Finance, 18(2), 265-282.

Giesecke, K. and Kim, B. (2011) : Risk Analysis of Collateralized Debt Obligations, Operations Research, 59(1), 32-49.

Hawkes, A. G. (1971a) : Point spectra of some mutually exciting point processes, Journal of the Royal Statistical Society. Series B (Methodological ) 33 (3), 438443.

Hawkes, A. G. (1971b) : Spectra of some self-exciting and mutually exciting point processes, Biometrika, 58(1), 83-90. 
Hawkes, A. G. and Oakes, D. (1974) : A cluster process representation of a self-exciting process, Journal of Applied Probability, 11, 493-503.

Herath, H. S. B. and Herath, T. S. (2011) : Copula Based Actuarial Model for Pricing Cyber-Insurance Policies. Insurance Markets and Companies: Analyses and Actuarial Computations, 2 (1). 7-20.

Jang, J. and Dassios, A. (2013) : A Bivariate Shot Noise Self-Exciting Process for Insurance, Insurance: Mathematics \& Economics, 53/3, 524-532.

Lu, X. and Abergel, F. (2018): High-dimensional Hawkes processes for limit order books: modelling, empirical analysis and numerical calibration, Quantitative Finance, 18(20), 249-264.

McNeil, A. J., Frey, R. and Embrechts, P. (2005): Quantitative Risk Management: Concepts, Techniques and Tools, Princeton University Press, USA.

Mukhopadhyay, A., Chatterjee, S., Saha, D., Mahanti, A. and Sadhukhan, S. K. (2006) : e-Risk management with insurance: A framework using copula aided Bayesian belief networks. In Proceedings of the 39th Annual Hawaii International Conference on System Sciences (HICSS'06), vol. 6, 126.1-126.6. Hoboken, NJ: IEEE.

Rambaldi, M., Bacry, E. and Lillo, F. (2017) : The role of volume in order book dynamics: a multivariate Hawkes process analysis, Quantitative Finance, 17(7), 999-1020.

Stabile, G. and Torrisi, G. L. (2010) : Risk processes with non-stationary Hawkes claims arrivals, Methodology and Computing in Applied Probability, 12(3), 415429 .

Xu, M. and Hua, L. (2017) : Cybersecurity Insurance: Modeling and Pricing, Society of Actuaries. Schaumburg, Illinois.

Yang, S. Y., Liu, A., Chen, J. and Hawkes, A. (2018) : Applications of a multivariate Hawkes process to joint modeling of sentiment and market return events, Quantitative Finance, 18(2), 295-310.

\section{Appendix}

\section{A Proof of Theorem 4.1}

Setting $\mathcal{A} f\left(\lambda^{(1)}, n^{(1)}, l^{(1)}, \lambda^{(2)}, n^{(2)}, l^{(2)}, t\right)=l^{(1)}$ in $(2.3)$, we have

$$
\mathcal{A} l^{(1)}=\mu_{1_{J}} \lambda^{(1)}
$$

As $L_{t}^{(1)}-L_{0}^{(1)}-\int_{0}^{t} \mathcal{A} l_{s}^{(1)} d s$ is a $\Im$-martingale, we have

$$
E\left\{L_{t}^{(1)}-\int_{0}^{t} \mathcal{A} l_{s}^{(1)} d s \mid \lambda_{0}^{(1)}\right\}=L_{0}^{(1)}
$$


Hence

$$
E\left(L_{t}^{(1)} \mid \lambda_{0}^{(1)}\right)=L_{0}^{(1)}+E\left\{\int_{0}^{t} \mathcal{A} l_{s}^{(1)} d s \mid \lambda_{0}^{(1)}\right\}=L_{0}^{(1)}+\mu_{1_{J}} \int_{0}^{t} E\left(\lambda_{s}^{(1)} \mid \lambda_{0}^{(1)}\right) d s
$$

and (4.16) and (4.17) follow using (4.1) and (4.2) in Proposition 4.1. Similarly, (4.18) and (4.19) can be obtained.

\section{B Proof of Corollary 4.1}

From the proof in Theorem 4.1, we have

$$
E\left(L_{t}^{(1)}-L_{0}^{(1)} \mid \lambda_{0}^{(1)}\right)=\mu_{1_{J}} \int_{0}^{t} E\left(\lambda_{s}^{(1)} \mid \lambda_{0}^{(1)}\right) d s
$$

and also we know $E\left(\lambda_{t}^{(1)}\right)$ from (4.5), then by assuming that $L_{0}^{(1)}=0$, we have

$$
E\left(L_{t}^{(1)}\right)=E\left(L_{t}^{(1)}-L_{0}^{(1)}\right)=\mu_{1_{J}} \int_{0}^{t} E\left(\lambda_{s}^{(1)}\right) d s=\mu_{1_{J}}\left(\frac{\mu_{1_{F_{1}}} \rho+a^{(1)} \delta^{(1)}}{\delta^{(1)}-\mu_{1_{G}}}\right) t .
$$

Similarly, we have

$$
E\left(L_{t}^{(2)}\right)=E\left(L_{t}^{(2)}-L_{0}^{(2)}\right)=\mu_{1_{K}} \int_{0}^{t} E\left(\lambda_{s}^{(2)}\right) d s=\mu_{1_{K}}\left(\frac{\mu_{1_{F_{2}}} \rho+a^{(2)} \delta^{(2)}}{\delta^{(2)}-\mu_{1_{H}}}\right) t
$$

\section{Proof of Lemma 4.1}

Setting $\mathcal{A} f\left(\Lambda^{(1)}, \lambda^{(1)}, n^{(1)}, l^{(1)}, \Lambda^{(2)}, \lambda^{(2)}, n^{(2)}, l^{(2)}, t\right)=\lambda^{(1)} l^{(2)}$ in $(2.3)$, we have

$$
\mathcal{A}\left(\lambda^{(1)} l^{(2)}\right)=-\left(\delta^{(1)}-\mu_{1_{G}}\right) \lambda^{(1)} l^{(2)}+\left(a^{(1)} \delta^{(1)}+\mu_{1_{F_{1}}} \rho\right) l^{(2)}+\mu_{1_{K}} \lambda^{(1)} \lambda^{(2)} .
$$

As $\lambda_{t}^{(1)} L_{t}^{(2)}-\lambda_{0}^{(1)} L_{0}^{(2)}-\int_{0}^{t} \mathcal{A}\left(\lambda_{s}^{(1)} L_{s}^{(2)}\right) d s$ is a $\Im$-martingale, given $L_{0}^{(2)}=0$ we have the ODE

$\frac{d E\left(\lambda_{t}^{(1)} L_{t}^{(2)}\right)}{d t}=-\left(\delta^{(1)}-\mu_{1_{G}}\right) E\left(\lambda_{t}^{(1)} L_{t}^{(2)}\right)+\left(a^{(1)} \delta^{(1)}+\mu_{1_{F_{1}}} \rho\right) E\left(L_{t}^{(2)}\right)+\mu_{1_{K}} E\left(\lambda_{t}^{(1)} \lambda_{t}^{(2)}\right)$

with the initial condition $E\left(\lambda_{0}^{(1)} L_{0}^{(2)}\right)=0$. The solution of this ODE using (4.21) and (4.9) is given by (4.22). Similarly, we have (4.23).

\section{Proof of Theorem 4.2}


Setting $\mathcal{A} f\left(\Lambda^{(1)}, \lambda^{(1)}, n^{(1)}, l^{(1)}, \Lambda^{(2)}, \lambda^{(2)}, n^{(2)}, l^{(2)}, t\right)=l^{(1)} l^{(2)}$ in $(2.3)$, we have

$$
\mathcal{A}\left(l^{(1)} l^{(2)}\right)=\mu_{1_{J}} \lambda^{(1)} l^{(2)}+\mu_{1_{K}} \lambda^{(2)} l^{(1)}
$$

As $L_{t}^{(1)} L_{t}^{(2)}-L_{0}^{(1)} L_{0}^{(2)}-\int_{0}^{t} \mathcal{A}\left(L_{s}^{(1)} L_{s}^{(2)}\right) d s$ is a $\Im$-martingale, we have

$$
E\left\{L_{t}^{(1)} L_{t}^{(2)}-\int_{0}^{t} \mathcal{A}\left(L_{s}^{(1)} L_{s}^{(2)}\right) d s \mid \lambda_{0}^{(1)}, \lambda_{0}^{(2)}\right\}=L_{0}^{(1)} L_{0}^{(2)}
$$

Hence

$$
\begin{gathered}
E\left(L_{t}^{(1)} L_{t}^{(2)} \mid \lambda_{0}^{(1)}, \lambda_{0}^{(2)}\right)=L_{0}^{(1)} L_{0}^{(2)}+E\left\{\int_{0}^{t} \mathcal{A}\left(L_{s}^{(1)} L_{s}^{(2)}\right) d s \mid \lambda_{0}^{(1)}, \lambda_{0}^{(2)}\right\} \\
=L_{0}^{(1)} L_{0}^{(2)}+\mu_{1_{J}} \int_{0}^{t} E\left(\lambda_{s}^{(1)} L_{s}^{(2)} \mid \lambda_{0}^{(1)}, \lambda_{0}^{(2)}\right) d s+\mu_{1_{K}} \int_{0}^{t} E\left(\lambda_{s}^{(2)} L_{s}^{(1)} \mid \lambda_{0}^{(1)}, \lambda_{0}^{(2)}\right) d s .
\end{gathered}
$$

Using (4.22) and (4.23), with $L_{0}^{(1)}=L_{0}^{(2)}=0$, we have

$$
E\left(L_{t}^{(1)} L_{t}^{(2)}\right)=\mu_{1_{J}} \int_{0}^{t} E\left(\lambda_{s}^{(1)} L_{s}^{(2)}\right) d s+\mu_{1_{K}} \int_{0}^{t} E\left(\lambda_{s}^{(2)} L_{s}^{(1)}\right) d s
$$

and the result follows.

\section{E Proof of Lemma 4.2}

Setting $\mathcal{A} f\left(\Lambda^{(1)}, \lambda^{(1)}, n^{(1)}, l^{(1)}, \Lambda^{(2)}, \lambda^{(2)}, n^{(2)}, l^{(2)}, t\right)=\lambda^{(1)} l^{(1)}$ in $(2.3)$, we have

$$
\mathcal{A}\left(\lambda^{(1)} l^{(1)}\right)=-\left(\delta^{(1)}-\mu_{1_{G}}\right) \lambda^{(1)} l^{(1)}+\left(a^{(1)} \delta^{(1)}+\mu_{1_{F_{1}}} \rho\right) l^{(1)}+\mu_{1_{J}}\left\{\lambda^{(1)}\right\}^{2}+\mu_{1_{G}} \mu_{1_{J}} \lambda^{(1)} .
$$
ODE,

As $\lambda_{t}^{(1)} L_{t}^{(1)}-\lambda_{0}^{(1)} L_{0}^{(1)}-\int_{0}^{t} \mathcal{A}\left(\lambda_{s}^{(1)} L_{s}^{(1)}\right) d s$ is a $\Im$-martingale, given $L_{0}^{(1)}=0$ we have the

$$
\begin{aligned}
& \frac{d E\left(\lambda_{t}^{(1)} L_{t}^{(1)}\right)}{d t}=-\left(\delta^{(1)}-\mu_{1_{G}}\right) E\left(\lambda_{t}^{(1)} L_{t}^{(1)}\right)+\left(a^{(1)} \delta^{(1)}+\mu_{1_{F_{1}}} \rho\right) E\left(L_{t}^{(1)}\right) \\
& +\mu_{1_{J}} E\left[\left\{\lambda_{t}^{(1)}\right\}^{2}\right]+\mu_{1_{G}} \mu_{1_{J}} E\left(\lambda_{t}^{(1)}\right) .
\end{aligned}
$$

with the initial condition $E\left(\lambda_{0}^{(1)} L_{0}^{(1)}\right)=0$. The solution of this ODE using (4.20), (4.14) and (4.5), is given by (4.27). Similarly, we have (4.28). 


\section{F Proof of Theorem 4.3}

Setting $\mathcal{A} f\left(\Lambda^{(1)}, \lambda^{(1)}, n^{(1)}, l^{(1)}, \Lambda^{(2)}, \lambda^{(2)}, n^{(2)}, l^{(2)}, t\right)=\left\{l^{(1)}\right\}^{2}$ in $(2.3)$, we have

$$
\mathcal{A}\left\{l^{(1)}\right\}^{2}=2 \mu_{1_{J}} \lambda^{(1)} l^{(1)}+\mu_{2_{J}} \lambda^{(1)} \text {. }
$$

As $\left\{L_{t}^{(1)}\right\}^{2}-\left\{L_{0}^{(1)}\right\}^{2}-\int_{0}^{t} \mathcal{A}\left\{L_{s}^{(1)}\right\}^{2} d s$ is a $\Im$-martingale, given $L_{0}^{(1)}=0$ we have

$$
E\left[\left\{L_{t}^{(1)}\right\}^{2}\right]=2 \mu_{1_{J}} \int_{0}^{t} E\left(\lambda_{s}^{(1)} L_{s}^{(1)}\right) d s+\mu_{2_{J}} \int_{0}^{t} E\left(\lambda_{s}^{(1)}\right) d s
$$

and (4.29) follows using (4.27) and (4.5). Similarly, we have (4.30).

\section{G Proof of Corollary 4.2}

By $\operatorname{Var}\left\{L_{t}^{(1)}\right\}=E\left[\left\{L_{t}^{(1)}\right\}^{2}\right]-\left\{E\left(L_{t}^{(1)}\right)\right\}^{2}$ and using (4.29) and (4.20), (4.31) follows. Similarly, we have (4.32). 